Pacific Journal of Mathematics

APPROXIMATING CODIMENSION TWO EMBEDDINGS OF 


\title{
APPROXIMATING CODIMENSION TWO EMBEDDINGS OF CELLS
}

\author{
Gerard A. Venema
}

\begin{abstract}
It is shown that every topological embedding of a $k$-cell into a piecewise linear $(k+2)$-manifold can be arbitrarily closely approximated by locally flat piecewise linear embeddings. The new ingredient in the proof is an $\varepsilon$-controlled piping lemma.
\end{abstract}

Introduction. The main result of this paper is the following.

THEOREM 1. Let $h: D^{k} \rightarrow M^{k+2}$ be a topological embedding of the $k$-cell $D^{k}$ into a piecewise linear $(k+2)$-manifold $M^{k+2}$. Then for every $\varepsilon>0$ there exists a locally flat piecewise linear embedding $g: D^{k} \rightarrow M^{k+2}$ such that $d(h(x), g(x))<\varepsilon$ for each $x \in D^{k}$.

Approximation theorems of this kind are already known for embeddings of cells in codimensions $\geq 3$ [2] and in case $k=2$ [4]. In addition, the case $k=3$ was announced in [6]. Here the techniques of [2] and [4] are combined to obtain Theorem 1. By [4, Corollary 1], it is enough to consider only the case $M=R^{k+2}$ and so that is the only case which will be mentioned in the remainder of this paper.

The proof given in this paper is modelled on Miller's proof in [2]. We must add a new ingredient to push the technique up to codimension two, but our proof does not give a new proof of Miller's result; it would reduce to exactly his proof in codimension three. The new proof is somewhat different from that in [4] in the case $k=2$.

The new ingredient needed to win the extra dimension is an $\varepsilon$-controlled piping lemma. It is commonly known that piping (in the sense of Zeeman [7, Lemma 48]) requires a global rather than local move; e.g., see the Remark in [7], Chapter VII, p. 45. However, in the presence of the topological embedding $h$, we are able to achieve that global modification by means of a homotopy which moves each point only a small distance. That requires more elaborate geometric constructions than those used by Zeeman. The geometry of the two kinds of piping is described at the beginning of $\S 2$. 
There are actually two piping lemmas in this paper. The first is an $\varepsilon$-controlled version of Zeeman's Lemmas 48 and 49 . The second involves piping more like that used by Zeeman in the proof of his unknotting theorem (see [7], Chapter VIII, Lemma 65). Since the point of these lemmas is that $\varepsilon$-control can be added to Zeeman's piping technique, we spell out very carefully in the proof of Lemma 1 just how all the $\varepsilon$ 's and $\delta$ 's work.

Recently Montejano [3] has generalized Miller's technique and stated the approximation theorem in terms of liftings. The same could be done here.

There are many open questions remaining in the study of topological embeddings in codimension two. In particular, it is not known whether $D^{k}$ in Theorem 1 can be replaced by some other $k$-manifold (such as $S^{k}$ or a $k$-manifold with only handles of index $\leq 1$, etc.).

Finally, I would like to thank Mike Starbird for explaining [2] to me in an unusually clear and beautiful way. I also wish to thank Luis Montejano for listening patiently to the proofs of the piping lemmas and for offering many helpful suggestions on how those proofs should be written. Thanks also to the referee for pointing out several inconsistencies in the first write-up of the proof of Lemma 3.

1. Preliminaries. We begin with some definitions and notation, most of which are found in [2] and [7]. The reader is warned, however, that there are minor differences between the terminology used here and that in [2]. We use [7] as a general reference for PL topology.

For each positive integer $k$, let $R^{k}$ denote $k$-dimensional Euclidean space and let $d$ be the usual distance function for $R^{k}$. If $X \subset R^{k}$ and $\varepsilon>0$, then $N_{\varepsilon}(X)=\left\{y \in R^{k} \mid d(y, X)<\varepsilon\right\}$. Let $I=[0,1]$. The $k$-cell $D^{k}$ is defined by $D^{k}=\left\{\left(x_{1}, \ldots, x_{k}\right) \in R^{k} \mid x_{i} \in I\right.$ for each $\left.i\right\}$. For $1 \leq j$ $\leq k$ and $0 \leq a \leq b \leq 1$, we define $D_{j}^{k}[a, b]$ to be $\left\{\left(x_{1}, \ldots, x_{k}\right) \in D^{k} \mid a\right.$ $\leq x, \leq b\}$ and $D_{J}^{k}[a]$ to be $D_{j}^{k}[a, a]$.

In the rest of this paper, assume that $k$ is a fixed positive integer and that a topological embedding $h: D^{k} \rightarrow R^{k+2}$ has been given.

For each $j, 1 \leq j \leq k$, we define two homotopies. First $\theta_{t}^{j}: D^{k} \rightarrow D^{k}$, $0 \leq t \leq 1$, is the deformation retraction defined by

$$
\theta_{t}^{j}\left(x_{1}, \ldots, x_{k}\right)=\left\{\begin{array}{l}
\left(x_{1}, \ldots, x_{k}\right) \text { if } x_{j} \geq t, \text { and } \\
\left(x_{1}, \ldots, x_{J-1}, t, x_{j+1}, \ldots, x_{k}\right) \text { if } x_{j} \leq t .
\end{array}\right.
$$

Notice that $\theta_{t}^{j}$ deforms $D^{k}$ to its $j$ th face, $D_{j}^{k}[1]$. We use the notations $\boldsymbol{\theta}_{t}^{j}(x)$ and $\boldsymbol{\theta}^{J}(x, t)$ interchangeably. 
As in [2], the fact that $h\left(D^{k}\right)$ is an ANR can be used to find a neighborhood $N$ of $h\left(D^{k}\right)$ and a retraction $r: N \rightarrow h\left(D^{k}\right)$. We may assume that $N \subset N_{1}\left(h\left(D^{k}\right)\right)$. Define $r_{t}(x)$ to be the homotopy which moves $x$ at constant speed along the straight line from $x$ to $r(x)$ during the time interval $0 \leq t \leq d(x, r(x))$ and remains fixed for other values of $t$. Now define a second homotopy, $\psi_{t}^{j}: N \rightarrow R^{k+2}, 0 \leq t \leq 1$, by

$$
\psi_{t}^{j}(x)=\left\{\begin{array}{l}
r_{t}(x) \quad \text { if } 0 \leq t \leq d(x, r(x)) \\
h \theta^{j}\left(h^{-1} r(x), \frac{t-d(x, r(x))}{1-d(x, r(x))}\right) \quad \text { if } d(x, r(x)) \leq t \leq 1 .
\end{array}\right.
$$

The important feature of $\psi_{t}^{j}$ is that $\psi_{t}^{j}$ extends $h \theta_{t}^{j} h^{-1}$ to all of $N$.

Suppose $X$ and $Y$ are polyhedra and that $X$ simplically collapses to $Y$ via a collapse $\xi$. (Write $\xi: X \searrow Y$.) We think of $\xi$ as specifying not only which elementary simplicial collapses are to be done, but also the timing of those collapses during the time interval $0 \leq t \leq 1$. The collapse $\xi$ then induces a strong deformation retraction $\xi_{t}$ of $X$ to $Y$ in a natural way (see [2], p. 408). Given $\xi: X \searrow Y$ and a subpolyhedron $Z$ of $X$, we will consider the following subpolyhedron of $X$ : $\operatorname{Trail}_{\xi}(Z)=\xi(Z \times I)$.

Let $f: K \rightarrow R^{n}$ be a piecewise linear map. The singular set of $f$ is defined by $S(f)=\overline{\left\{y \in K \mid f^{-1} f(y) \neq\{y\}\right\}}$. Observe that if $\xi: K \searrow L$ is a collapse, then $f(K) \searrow f(L) \cup f\left(\right.$ Trail $\left._{\xi} S(f)\right)$. The support of a map $f$ is defined by $\operatorname{supp}(f)=\{x \mid f(x) \neq x\}$.

2. The first piping lemma. In this section we prove an $\varepsilon$-controlled version of Zeeman's Piping Lemma. Piping is a technique in which part of the singular set of a homotopy is pushed off the edge of the track of the homotopy. The important consequence is that a hole is punched in each top dimensional simplex of the singular set, allowing us to collapse out things below that simplex. Figure 0 illustrates the usual Zeeman Piping procedure.

The usual procedure is not good enough for our purposes here because the pipe must, in general, be very long and thus the modification moves some points too far. We will describe a more elaborate procedure which produces the same holes in the singular set but does so without moving any point very far. The reader can refer ahead to Figures 4 and 5 for pictures of the kind of modification we will make. Since the horizontal distance in Figure 4 can be made arbitrarily small, no point is moved very far by the push pictured there. 


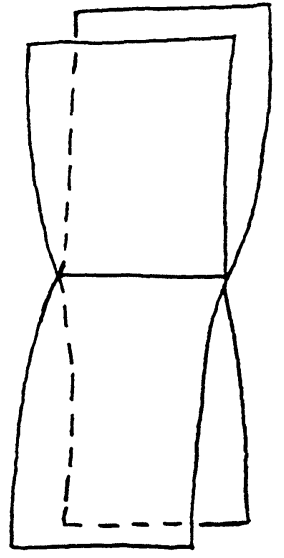

Before (range)

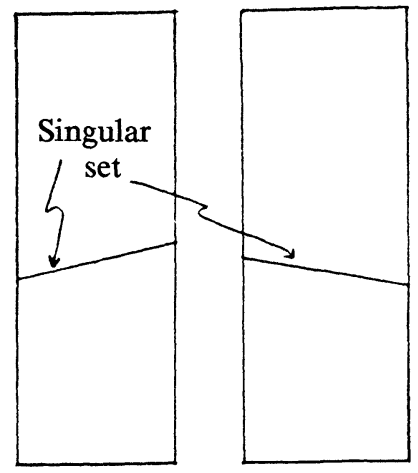

Before (domain)

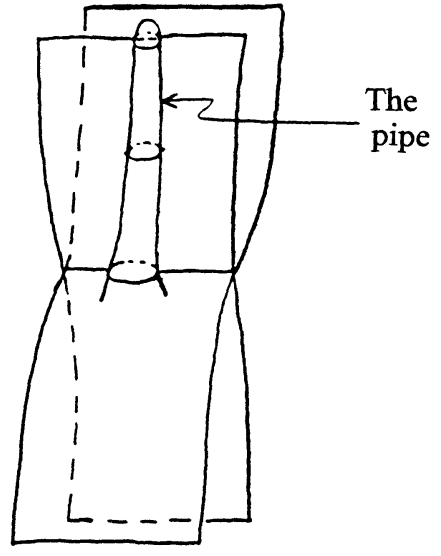

After (range)
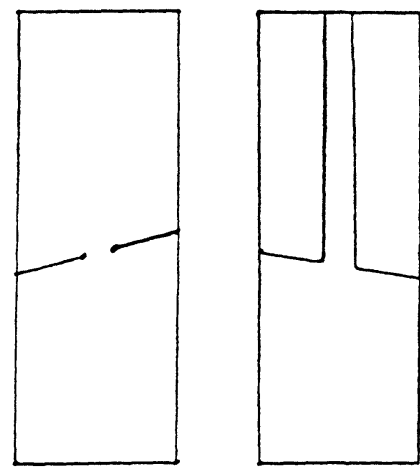

After (domain)

\section{FIGURE 0}

The reader is warned that the proof of Lemma 1 should be understood thoroughly before the proofs of the subsequent lemmas are attempted. All the details of the epsilonics are spelled out in the proof of Lemma 1 but are left out of the proofs of the later lemmas whenever they are essentially the same as those in earlier proofs.

We begin with two more definitions.

As stated above, the positive integer $k$ and the topological embedding $h: D^{k} \rightarrow R^{k+2}$ are fixed. For each $x \in h\left(D^{k}\right)$ and $1 \leq j \leq k$, we define the fiber through $x$ in the jth direction to be $F_{j}(x)=\left\{y \in h\left(D^{k}\right) \mid h^{-1}(x)\right.$ and $h^{-1}(y)$ differ in at most their $j$ th coordinates $\}$.

Suppose $Y$ is a polyhedron in $N, Z$ and $C$ are subpolyhedra of $Y$ and $\varepsilon>0$. We say that a collapse $\xi: Y \searrow Z \cup C$ is a $(j, \varepsilon, Z)$-collapse if

(a) $C \subset N_{\varepsilon}\left(h\left(D_{j}^{k}[1]\right)\right)$,

(b) $\xi_{t}(Y) \subset N_{\varepsilon}\left(h\left(D_{j}^{k}[t, 1]\right)\right) \cup Z$ for all $t \in I$, 
(c) $\operatorname{supp} \xi_{t} \subset N_{\varepsilon}\left(h\left(D_{j}^{k}[0, t]\right)\right)$ for all $t \in I$, and

(d) for each $y \in Y$ there exists one $x \in h\left(D^{k}\right)$ such that $\xi_{t}(y) \in$ $N_{\varepsilon}\left(F_{j}(x)\right)$ for all $t \in I$.

LEMMA 1. For every $\varepsilon>0$ and $1 \leq j \leq k$ there exists $a \delta>0$ such that if $X$ is a compact polyhedron in $N_{\delta}\left(h\left(D_{j}^{k}[0]\right)\right)$ with $\operatorname{dim} X=k-1$, then there exists a PL map $f: X \times I \rightarrow N_{\varepsilon}\left(h\left(D^{k}\right)\right)$ and a subpolyhedron $J_{0}$ of $X \times I$ such that

(1) $f(x, 0)=x$ for each $x \in X$,

(2) $f(x, t) \in N_{\varepsilon}\left(h\left(D_{j}^{k}[t]\right)\right) \cap N_{\varepsilon}\left(F_{j}(r(x))\right)$ for each $(x, t) \in X \times I$,

(3) $\operatorname{dim} J_{0} \leq k-2$, and

(4) there is a $\left(j, \varepsilon, f\left(J_{0}\right)\right)$-collapse $\xi: f(X \times I) \searrow f(X \times\{1\}) \cup f\left(J_{0}\right)$.

Proof. The lemma is trivial for $k \leq 1$, so we assume that $k \geq 2$. Begin by choosing a number $\alpha>0$ such that if $t_{1}, t_{2} \in I$ and $\left|t_{1}-t_{2}\right|<\alpha$, then $h\left(D_{j}^{k}\left[t_{1}\right]\right) \subset N_{\varepsilon / 3}\left(h\left(D_{j}^{k}\left[t_{2}\right]\right)\right)$. Let $0=t_{0}<t_{1}<t_{2}<\cdots<t_{n}=1$ be a partition of $[0,1]$ with $\left|t_{i}-t_{i-1}\right|<\alpha$ for each $i$. Choose $\beta>0$ such that if $A$ is any subset of $N \times I$ of diameter $<\beta$, then $\operatorname{diam} \psi^{j}(A)<\varepsilon / 3$.

The most important conclusion of the lemma is (3). To illustrate that point, we quickly choose a number $\delta$ and use it to get conclusions (1), (2), and (4) as stated. However, in this first attempt we are off by one dimension from achieving conclusion (3). The remainder of the proof then consists of showing how to choose $\delta$ more carefully and how to modify the easy first attempt to lower the dimension of $J_{0}$ by 1 and get conclusion (3) as well.

Choose $\delta>0$ so small that $\psi_{t}^{j}(x) \in N_{\varepsilon / 3}\left(h\left(D_{j}^{k}[t]\right)\right)$ and $d(x, r(x))$ $<\varepsilon / 3$ for every $x \in N_{\delta}\left(h\left(D_{j}^{k}[0]\right)\right)$ and for every $t \in I$. Suppose $X \subset$ $N_{\delta}\left(h\left(D_{j}^{k}[0]\right)\right)$ is a compact polyhedron of dimension $k-1$. Let $f: X \times I$ $\rightarrow N_{\varepsilon}\left(h\left(D^{k}\right)\right)$ be a PL, general position ( $\left.\varepsilon / 3\right)$-approximation to $\psi^{j} \mid X \times$ $I$, keeping $X \times\{0\}$ fixed. Conclusions (1) and (2) of the Lemma are then obvious.

Triangulate $X \times I$ with a cylindrical triangulation which has mesh less than $\beta$ and includes each of the sets $X \times\left\{t_{i}\right\}, i=0,1, \ldots, n$ and $S(f)$ as subcomplexes. Let $\mu: X \times I \searrow X \times\{1\}$ be a cylindrical collapse of that triangulation, timed so that all the simplices in $X \times\left[t_{i-1}, t_{i}\right]$ are collapsed out during the time interval $t_{i-1} \leq t \leq t_{i}$. Let $J_{0}=\operatorname{Trail}_{\mu}(S(f))$. Define $\xi: f(X \times I) \searrow f(X \times\{1\}) \cup f\left(J_{0}\right)$ to be the collapse induced by $\mu$.

We claim that $\xi$ is a $\left(j, \varepsilon, f\left(J_{0}\right)\right)$-collapse. It is necessary to check conditions (a)-(d). 
(a) $C=f(X \times\{1\})$ is contained in $N_{\varepsilon}\left(h\left(D_{j}^{k}[1]\right)\right)$ by the choice of $\delta$.

(b) Let $t \in[0,1]$. Then $t \in\left[t_{i-1}, t_{i}\right]$ for some $i$. Now $\mu_{t}(X \times I) \subset$ $X \times\left[t_{i-1}, 1\right]$, so

$$
\begin{aligned}
\xi_{t}(f(X \times I)) & \subset f\left(X \times\left[t_{i-1}, 1\right]\right) \cup f\left(J_{0}\right) \\
& \subset N_{\varepsilon / 3}\left(\psi^{j}\left(X \times\left[t_{i-1}, 1\right]\right)\right) \cup f\left(J_{0}\right) \\
& \subset N_{2 \varepsilon / 3}\left(h\left(D_{j}^{k}\left[t_{i-1}, 1\right]\right)\right) \cup f\left(J_{0}\right) \\
& \subset N_{\varepsilon}\left(h\left(D_{j}^{k}[t, 1]\right)\right) \cup f\left(J_{0}\right) .
\end{aligned}
$$

(c) Let $t \in[0,1]$. Again $t \in\left[t_{i-1}, t_{i}\right]$ for some $i$. Since supp $\mu_{t} \subset X \times$ $\left[0, t_{i}\right]$, we must have

$$
\begin{aligned}
\operatorname{supp}\left(\xi_{t}\right) & \subset f\left(X \times\left[0, t_{i}\right]\right) \\
& \subset N_{\varepsilon / 3}\left(\psi^{j}\left(X \times\left[0, t_{i}\right]\right)\right) \\
& \subset N_{2 \varepsilon / 3}\left(h\left(D_{j}^{k}\left[0, t_{i}\right]\right)\right) \\
& \subset N_{\varepsilon}\left(h\left(D_{j}^{k}[0, t]\right)\right) .
\end{aligned}
$$

(d) Let $y \in f(X \times I)$. Then $y=f(x, s)$ for some $(x, s) \in X \times I$. There is a simplex $\sigma$ of $X$ such that $x \in \sigma$. Because $\mu$ is a cylindrical collapse, $\xi_{t}(y) \in f(\sigma \times I)$ for every $t$. Thus

$$
\begin{aligned}
\xi_{t}(y) & \in f(\sigma \times I) \\
& \subset N_{\varepsilon / 3}\left(\psi^{j}(\sigma \times I)\right) \\
& \left.\subset N_{2 \varepsilon / 3}\left(\psi^{j}(\{x\} \times I)\right) \quad \text { (by the choice of } \beta\right) \\
& \subset N_{\varepsilon}\left(F_{j}(r(x))\right) .
\end{aligned}
$$

Hence $f, J_{0}$ satisfy all the conclusions of the Lemma except (3). The best we can hope for is that, for $J_{0}$ as defined above,

$$
\begin{aligned}
\operatorname{dim} J_{0} & \leq \operatorname{dim} S(f)+1 \\
& \leq 2[(k-1)+1]-(k+2)+1=k-1 .
\end{aligned}
$$

As is usual in a piping argument, we want to homotope $f$ around in such a way that a hole is punched in each top-dimensional simplex of $S(f)$, allowing us to collapse out what is below that simplex without running into $S(f)$. That will require a more complicated choice of $\delta$.

The choice of $\delta$.

We choose $\delta$ inductively. 
First choose $\delta_{0}$ so that $0<\delta_{0} \leq \varepsilon / 3$ and $N_{\delta_{0}}\left(h\left(D_{j}^{k}\left[0, t_{i-1}\right]\right)\right) \cap$ $N_{\delta_{0}}\left(h\left(D_{j}^{k}\left[t_{i}, 1\right]\right)\right)=\varnothing$ for each $i=1, \ldots, n$.

Next choose $\delta_{1}, 0<\delta_{1} \leq \delta_{0}$, such that if $x_{1}, x_{2} \in h\left(D^{k}\right)$ and $N_{\delta_{1}}\left(F_{j}\left(x_{1}\right)\right) \cap N_{\delta_{1}}\left(F_{j}\left(x_{2}\right)\right) \neq \varnothing$, then $N_{\delta_{1}}\left(F_{j}\left(x_{1}\right)\right) \subset N_{\delta_{0}}\left(F_{j}\left(x_{2}\right)\right)$. (The existence of $\delta_{1}$ follows from the uniform continuity of $h$ and $h^{-1}$.)

Now for the induction. Suppose $\delta_{0}, \delta_{1}, \ldots, \delta_{i}$ have been chosen, $1 \leq i \leq n-1$. Choose numbers $s_{i}^{i}, s_{i+1}^{i}, \ldots, s_{n-1}^{i}$ such that $t_{i}<s_{i}^{i}<t_{t+1}$ $<s_{i+1}^{i}<\cdots<t_{n-1}<s_{n-1}^{l}<t_{n}=1$ and such that $h\left(D_{j}^{k}\left[t_{q}, s_{q}^{i}\right]\right) \subset$ $N_{\delta_{i}}\left(h\left(D_{j}^{k}\left[t_{q}\right]\right)\right)$ for $q=i, i+1, \ldots, n-1$. Let $\eta_{i} \leq \delta_{i}$ be a positive number so small that the sets $N_{\eta_{t}}\left(h\left(D_{j}^{k}\left(\left[0, t_{i}\right]\right)\right)\right.$ and $\left\{N_{\eta_{t}}\left(h\left(D_{j}^{k}\left[s_{q}^{i}, t_{q+1}\right]\right)\right)\right\}_{q=i}^{n-1}$ are pairwise disjoint. We also make $\eta_{t}$ small enough so that $N_{\eta_{t}}\left(h\left(D_{j}^{k}\left[t_{q}, s_{q}^{l}\right]\right)\right) \subset N_{\delta_{i}}\left(h\left(D_{j}^{k}\left[t_{q}\right]\right)\right)$ for $q=i, i+1, \ldots, n-1$. By [5, Corollary 2.2], there exists a positive number $\gamma_{i}$ with the following property: If $K$ is any compact, 1-dimensional polyhedron in $N_{\gamma_{t}}\left(h\left(D_{j}^{k}\left[s_{q}^{i}, t_{q+1}\right]\right)\right)$ for some $q \geq i$, then there is a PL ambient isotopy $H_{t}$ such that

(i) $H_{0}=$ id,

(ii) supp $H_{t} \subset N_{\eta_{t}}\left(h\left(D_{j}^{k}\left[s_{q}^{i}, t_{q+1}\right]\right)\right)-N_{\gamma_{t}}\left(h\left(D_{j}^{k}\left[0, s_{q}^{i}\right]\right)\right)$,

(iii) $H_{1}(K) \subset N_{\eta_{i}}\left(h\left(D_{j}^{k}\left[s_{q}^{i}\right]\right)\right)$, and

(iv) $H_{t}\left(N_{\gamma}\left(F_{j}(x)\right)\right) \subset N_{\eta_{t}}\left(F_{j}(x)\right)$ for every $t \in I$ and $x \in h\left(D^{k}\right)$. Moreover, there exists a PL map $g: K \times I \rightarrow N_{\eta_{t}}\left(h\left(D_{j}^{k}\left[s_{q}^{i}, t_{q+1}\right]\right)\right)$ such that $H_{t}$ can be chosen to have its support in an arbitrarily small neighborhood of $g(K \times I)$.

Now choose $\delta_{i+1}, 0<\delta_{i+1} \leq \gamma_{i}$ such that if $x_{1}, x_{2} \in y\left(D^{k}\right)$ and $N_{\delta_{t+1}}\left(F_{j}\left(x_{1}\right)\right) \cap N_{\delta_{t+1}}\left(F_{j}\left(x_{2}\right)\right) \neq \varnothing$, then $N_{\delta_{t+1}}\left(F_{j}\left(x_{1}\right)\right) \subset N_{\gamma_{t}}\left(F_{j}\left(x_{2}\right)\right)$.

After $\delta_{0}$ through $\delta_{n}$ have been chosen inductively, we finally choose $\delta>0$ such that $\psi_{t}^{j}(x) \in N_{\delta_{n}}\left(h\left(D_{j}^{k}[t]\right)\right) \cap N_{\delta_{n}}\left(F_{j}(r(x))\right)$ for each $x \in$ $N_{\delta}\left(h\left(D_{j}^{k}[0]\right)\right)$ and for every $t \in I$.

The first approximation of $f$.

Suppose $X$ is as in the statement of the lemma. Let $f_{n}: X \times I \rightarrow N$ be a PL, general position map which satisfies $f_{n}(x, 0)=x$ for each $x \in X$ and which so closely approximates $\psi^{j} \mid X \times I$ that $f_{n}(x, t) \in$ $N_{\delta_{n}}\left(h\left(D_{j}^{k}[t]\right)\right) \cap N_{\delta_{n}}\left(F_{j}(r(x))\right)$ for each $(x, t) \in X \times I$. Triangulate $X \times I$ with a cylindrical triangulation of mesh $<\beta$ which includes each of the sets $X \times\left\{t_{i}\right\}$ and $S\left(f_{n}\right)$ as subcomplexes. Let $\mu: X \times I \searrow X \times\{1\}$ be a cylindrical collapse, timed as before. This time, let $S=S\left(f_{n}\right)$ and let $J_{0}=\operatorname{Trail}_{\mu}\left(S^{(k-3)}\right)$ where $S^{(k-3)}$ denotes the $(k-3)$-dimensional skeleton of $S$. Notice that $\operatorname{dim} J_{0} \leq(k-3)+1=k-2$. We hope to homotope $f_{n}$, keeping the image of $J_{0} \cup(X \times\{0,1\})$ fixed, to a map $f_{0}$ with the property that $f_{0}(X \times I) \searrow f_{0}(X \times\{1\}) \cup f_{0}\left(J_{0}\right)$. 
Let $\pi: X \times I \rightarrow X$ denote projection onto the first coordinate. We may assume that if $\sigma$ is a top-dimensional simplex in $S$, then $\pi \sigma \cap$ $\pi(\overline{S-\sigma}) \subset \pi(\partial \sigma)$ [7, Chapter VII, Sublemma 1]. It may also be assumed that each top-dimensional simplex $\sigma$ of $S$ is horizontal in $X \times I$; i.e., $\pi \mid \sigma$ is a homeomorphism. (Those two assumptions will require slight modifications of the map $f_{n}$. The modifications are explaned in [1, Theorem 2.5].)

The $(k-2)$-dimensional simplices of $S$ will come in pairs, $\left\{\sigma_{1}, \sigma_{2}\right\}$, with the property that each simplex in a pair is embedded and the two have the same image. Thus $f_{n}\left(\sigma_{1}\right)=f_{n}\left(\sigma_{2}\right)$, but $f_{n}\left(\right.$ int $\left.\sigma_{i}\right) \cap f_{n}($ int $\sigma)=\phi$ for every $(k-2)$-simplex $\sigma$ in $S$ with $\sigma \neq \sigma_{1}$ and $\sigma \neq \sigma_{2}$.

\section{The two basic constructions.}

Let $\left\{\sigma_{1}, \sigma_{2}\right\}$ be a matched pair of $(k-2)$-simplices in $S$. The choice of $\delta_{0}$ guarantees that either there is an $i$ such that $\sigma_{1} \cup \sigma_{2} \subset X \times\left[t_{i-1}, t_{i}\right]$ or an $i$ such that $\sigma_{1} \subset X \times\left[t_{i-1}, t_{i}\right]$ and $\sigma_{2} \subset X \times\left[t_{i}, t_{i+1}\right]$ (relabel if necessary). There are two different modifications we might make, depending on which of the two cases occurs.

Case 1. $\sigma_{1} \cup \sigma_{2} \subset X \times\left[t_{i-1}, t_{i}\right]$. Following the proof of Zeeman [7, Lemma 48] exactly, pipe the image of $\sigma_{1}$ off the $t_{i-1}$ end of the image of $X \times\left[t_{i-1}, t_{i}\right]$. As long as $i>1$, the result is a new singular set which is the same as the old one except that $\sigma_{2}$ is replaced by a $(k-2)$-cell $\sigma_{2}^{\prime}$ which curves up over the $t_{i}$-level as indicated in Figure 1.
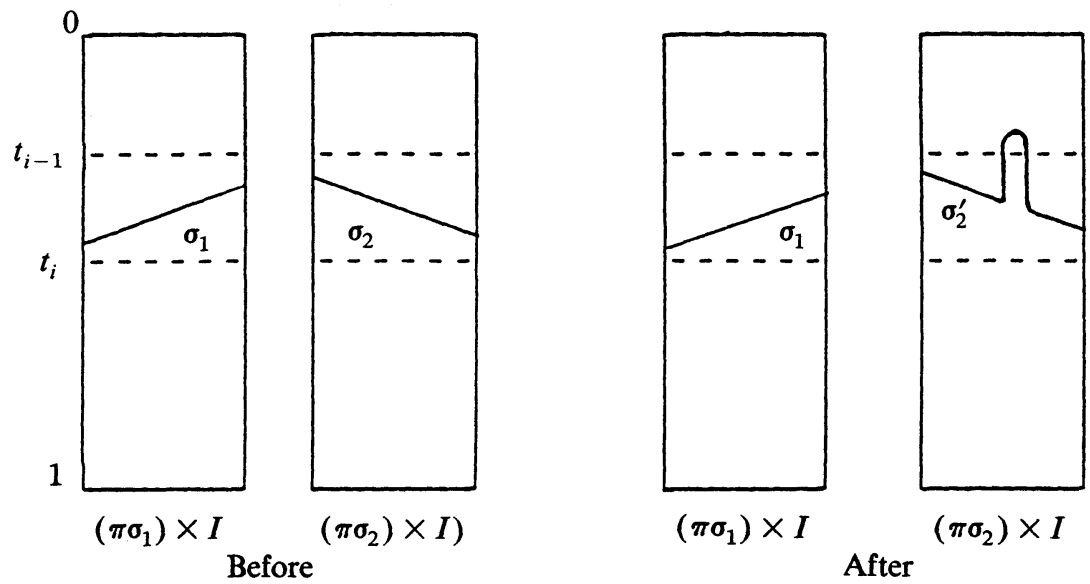

FIGURE 1

If $i=1$, we would actually push the image of the barycenter of $\sigma_{1}$ completely off the end of $f_{n}(X \times I)$. The picture would then be as indicated in Figure 2; we would have succeeded in punching the desired holes in $\sigma_{1}$ and $\sigma_{2}$. 

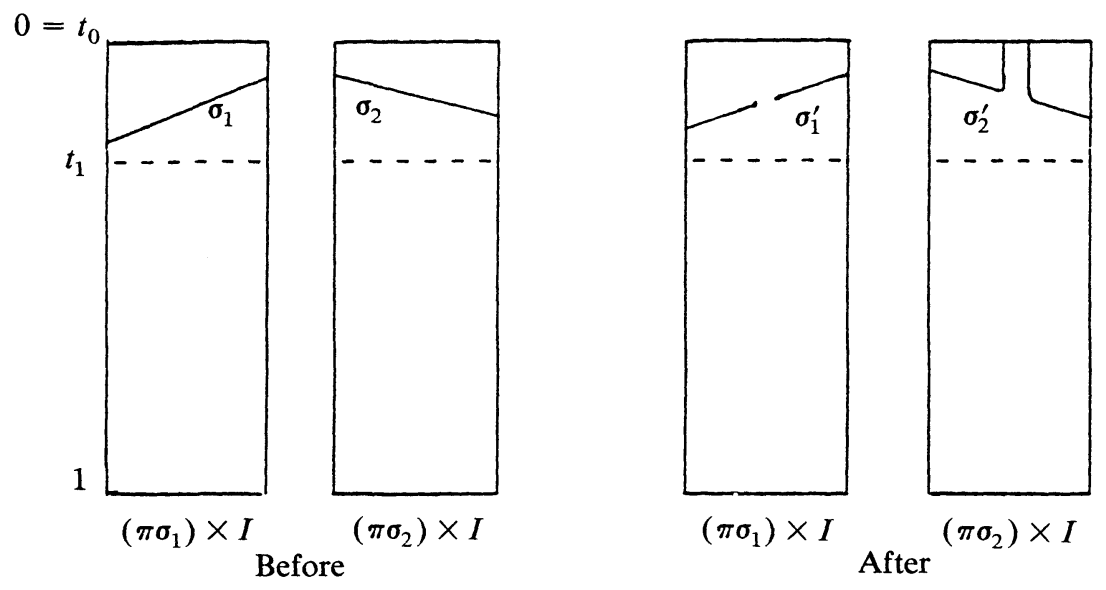

FIGURE 2

We will refer to the modification done in Case 1 as a "Type I Piping." A Type I piping is simply a Zeeman piping done to a small slice, $X \times\left[t_{i-1}, t_{i}\right]$, of $X \times I$.

Case 2. $\sigma_{1} \subset X \times\left[t_{i-1}, t_{i}\right], \sigma_{2} \subset X \times\left[t_{i}, t_{l+1}\right]$. Let $A$ be the vertical arc in $X \times I$ joining the barycenter of $\sigma_{2}$ to the level $X \times\left\{t_{i}\right\}$. Notice that both ends of $f_{n}(A)$ lie in $N_{\delta_{n}}\left(h\left(D_{j}^{k}\left[t_{i-1}, t_{l}\right]\right)\right)$. The objective is to push all of $f_{n}(A)$ near $h\left(D_{J}^{k}\left[t_{t-1}, t_{i}\right]\right)$ keeping the ends of $f_{n}(A)$ fixed. Suppose for the moment that that can be done and that $f_{n}^{\prime}$ is the map $f_{n}$ followed by such a push. A new map is defined which is composed of a vertical push straight down along $A$ in $X \times I$ followed by $f_{n}^{\prime}$. The new map has exactly the same image set as $f_{n}^{\prime}$; the only difference is in how that image is parametrized. Figure 3 shows what happens to the singular set. We will call this kind of modification a "Type II Piping."

The details of how we push $f_{n}(A)$ to $f_{n}^{\prime}(A)$ while still maintaining $\varepsilon$-control over where individual points go are exactly the same as those in the proofs of [4, Lemma 3] and [5, Lemma 3.2], but we give a condensed version here as well.

Let $K$ be a compact subplyhedron of $f_{n}(A)$ such that

$$
f_{n}(A)-N_{\delta_{i+1}}\left(h\left(D_{j}^{k}\left[t_{i}, s_{i}^{l}\right]\right)\right) \subset K \subset N_{\delta_{i+1}}\left(h\left(D_{J}^{k}\left[s_{i}^{l}, t_{i+1}\right]\right)\right) .
$$

By the choice of $\gamma_{i}$, there exists a PL map $g: K \times I \rightarrow N_{\eta_{i}}\left(h\left(D_{J}^{k}\left[s_{l}^{i}, t_{l+1}\right]\right)\right)$ such that $f_{n}(A)$ can be pushed into $N_{\eta_{t}}\left(h\left(D_{j}^{k}\left[t_{l-1}, t_{i}\right]\right)\right)$ with an ambient isotopy having support in an arbitrarily small neighborhood of $g(K \times I)$. What we have to watch out for is that the isotopy may pull some part of $f_{n}\left(X \times\left[t_{i+1}, t_{i+2}\right]\right)$ out of a neighborhood of $h\left(D_{j}^{k}\left[t_{i+1}, t_{i+2}\right]\right)$. Put $g$ in 


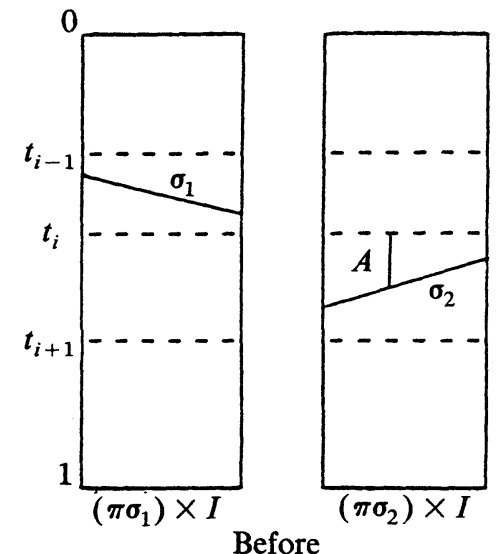

Before

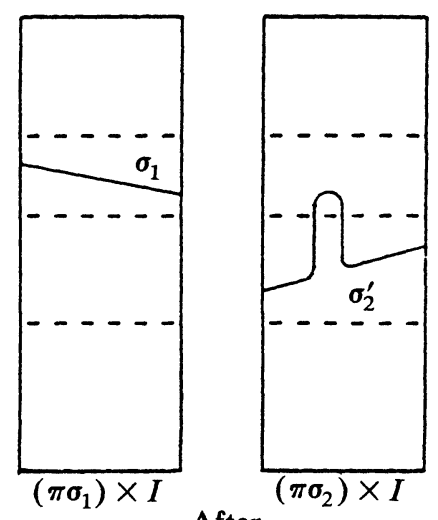

After

FIGURE 3

general position and consider $g(K \times I) \cap f_{n}\left(X \times\left[t_{i+1}, t_{i+2}\right]\right)$. That intersection consists of a finite number of points. Let $A_{1}$ be the union of a finite number of vertical arcs in $X \times I$ joining those points to $X \times\left\{t_{i+1}\right\}$. Associated with $A_{1}$ there is a compact 1-dimensional polyhedron $K_{1} \subset$ $f_{n}\left(A_{1}\right)$ and a PL map $g_{1}: K_{1} \times I \rightarrow N_{\eta_{t}}\left(h\left(D_{j}^{k}\left[s_{i+1}^{i}, t_{i+2}\right]\right)\right)$ such that $f_{n}\left(A_{1}\right)$ can be pulled near $h\left(D_{j}^{k}\left[t_{i}, t_{i+1}\right]\right)$ along $g_{1}\left(K_{1} \times I\right)$. Next consider $g_{1}\left(K_{1} \times I\right) \subset f_{n}\left(X \times\left[t_{i+2}, t_{i+3}\right]\right)$ - again a finite number of points. There will be a collection $A_{2}$ of vertical arcs associated with those points, a subset $K_{2} \subset f_{n}\left(A_{2}\right)$ and a PL map $g_{2}$ of $K_{2} \times I$, etc. The procedure is contained inductively down to $f_{n}\left(X \times\left[t_{n-1}, t_{n}\right]\right)$.

There will be arcs $A_{n-i-1}$, a subset $K_{n-i-1} \subset f_{n}\left(A_{n-i-1}\right)$ and a PL map $g_{n-t-1}: K_{n-i-1} \times I \rightarrow N_{\eta_{t}}\left(h\left(D_{j}^{k}\left[t_{n-1}, t_{n}\right]\right)\right)$. Push $f_{n}\left(A_{n-i-1}\right)$ across $g_{n-i-1}\left(K_{n-i-1} \times I\right)$ into a neighborhood of $h\left(D_{j}^{k}\left[t_{n-2}, t_{n-1}\right]\right)$. Then push the $t_{n-1}$-level of $X \times I$ straight down near $A_{n-i-1}$ so that $A_{n-1-1} \subset X \times$ $\left[t_{n-2}, t_{n-1}\right]$. Next push $f_{n}\left(A_{n-i-2}\right)$ across $g_{n-i-2}\left(K_{n-i-2} \times I\right)$ into a neighborhood of $h\left(D_{j}^{k}\left[t_{n-3}, t_{n-2}\right]\right)$ and then push the $t_{n-2}$-level of $X \times I$ down near $A_{n-i-2}$ until $A_{n-i-2} \subset X \times\left[t_{n-3}, t_{n-2}\right]$. This is continued back up the levels until eventually $A \subset X \times\left[t_{i-1}, t_{i}\right]$ and the Type II piping is complete.

Because the various ambient pushes have support on disjoint sets (by the choice of $\left.\eta_{i}\right)$ and because the fibers of $f_{n}(X \times I)$ are left setwise fixed by the vertical pushes in $X \times I$, the fibers $\left\{f_{n}(\{x\} \times I)\right\}$ are still where they should be. In addition, each slice $f_{n}\left(X \times\left[t_{q-1}, t_{q}\right]\right)$ is still near $h\left(D_{J}^{k}\left[t_{q-1}, t_{q}\right]\right)$, so we have the desired control over where individual points go. The details of those epsilonics are worked out in the proof of the claim below. 


\section{Construction of one pipe.}

Suppose again that $\sigma_{1}, \sigma_{2}$ are two $(k-2)$-simplices of $S$ which are identified under $f_{n}$. Assume for the moment also that $\sigma_{1} \cup \sigma_{2} \subset X \times$ $\left[t_{i-1}, t_{i}\right], i>1$. One Type I piping results in a new singular set which includes $\sigma_{1}$ but has a new $(k-2)$-cell $\sigma_{2}^{1}$ in place of $\sigma_{2}$. The boundary of $\sigma_{2}^{\prime}$ is in $X \times\left[t_{i-1}, t_{i}\right]$, but the center lies in $X \times\left[t_{i-2}, t_{i-1}\right]$. If we restrict our attention to small simplices $\sigma_{1}$ and $\sigma_{2}$ interior to $\sigma_{1}$ and $\sigma_{2}^{\prime}$, we have a pair which fits Case 2, above. We can do a Type II piping to that pair. In the centers of the resulting $(k-2)$-cells we could find another pair of cells, both of which are contained in $X \times\left[t_{i-2}, t_{i-1}\right]$. Go ahead and do a Type I piping to them, then a Type II, etc. Eventually we will find ourselves doing a Type $\mathrm{I}$ piping in $X \times\left[t_{0}, t_{1}\right]$. Stop after doing that move; the pipe is now constructed.

Figure 4 indicates the overall result of all of those moves in the range.

Figure 5 shows the overall result in the domain, $X \times I$.

If we have started in the situation $\sigma_{1} \subset X \times\left[t_{i-1}, t_{i}\right], \sigma_{2} \subset X \times$ $\left[t_{i}, t_{i+1}\right]$, we would just have had one extra Type II piping to do, but could still have achieved the same end result.

Since all the modifications involved in the moves described above are done in neighborhoods of 2-dimensional sets and $f_{n}\left(S \cup J_{0} \cup X \times\{0,1\}\right)$ has codimension 3, we can do all that and leave $f_{n} \mid\left(S-\sigma_{1} \cup \sigma_{2}\right) \cup J_{0} \cup$ $X \times\{0,1\}$ unchanged.

\section{Construction of all the pipes simultaneously.}

Rather than working on one pipe at a time, we actually construct them all at once as follows: First do all the Type I pipings in $X \times\left[t_{n-1}, t_{n}\right]$, then all the Type II pipings in $X \times\left[t_{n-2}, t_{n}\right]$, next all the Type I's in

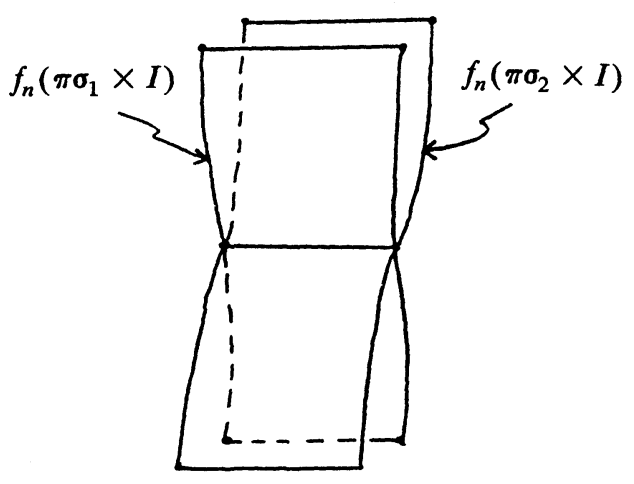

Before

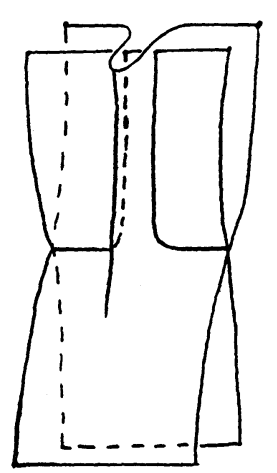

After 

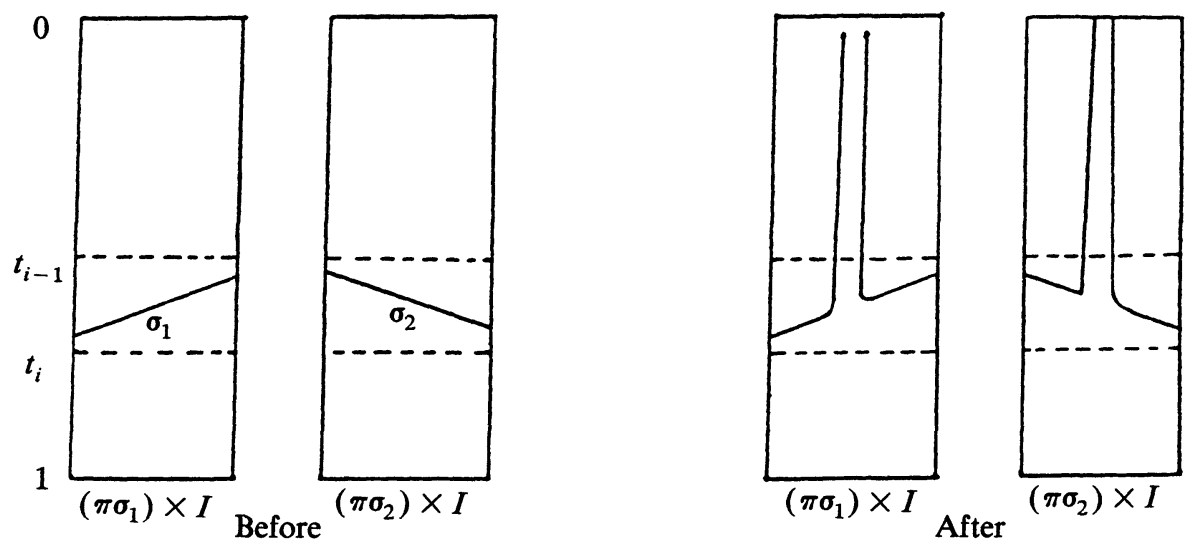

FIGURE 5

$X \times\left[t_{n-2}, t_{n-1}\right]$ and so on, alternating Type I and Type II pipings and ending with all the Type I pipings in $X \times\left[t_{0}, t_{1}\right]$.

Let $f_{n}^{\prime}$ denote the map which results from doing all the Type I pipings in $X \times\left[t_{n-1}, t_{n}\right]$ to $f_{n}$ and let $f_{n-1}$ denote the map which results from doing all the Type II pipings in $X \times\left[t_{n-2}, t_{n}\right]$ to $f_{n}^{\prime}$. In general, $f_{i}^{\prime}$ will denote the map which we have after all the Type I pipings in $X \times\left[t_{i-1}, t_{i}\right]$ have been done and $f_{i-1}$ will denote the map which results from doing all the Type II pipings in $X \times\left[t_{i-2}, t_{i}\right]$ to $f_{i}^{\prime}$. In each case $f_{i}$ is a PL map of $X \times I$ into $R^{k+2}$.

Claim. We can do the construction so carefully that each $f_{i}$ satisfies $f_{i}(\{x\} \times I) \subset N_{\delta_{i}}\left(F_{j}(r(x))\right)$ for every $x \in X$ and $f_{i}\left(X \times\left[t_{q-1}, t_{q}\right]\right) \subset$ $N_{\delta_{t}}\left(h\left(D_{j}^{k}\left[t_{q-1}, t_{q}\right]\right)\right)$ for every $q=1, \ldots, n$.

The proof of the claim is by downward induction on $i$. The case $i=n$ is obvious from the definition of $f_{n}$. So suppose the claim is true for some $f_{i}$. We get from $f_{i}$ to $f_{i-1}$ in two steps: we first do all the Type I pipings in $X \times\left[t_{i-1}, t_{i}\right]$ to get $f_{i}^{\prime}$ and then the Type II pipings in $X \times\left[t_{i-2}, t_{i}\right]$ to get $f_{i-1}$.

Now $f_{i}^{\prime}$ agrees with $f_{i}$ everywhere except near a finite number of points in $X \times\left(t_{i-1}, t_{i}\right)$. Each of those points is piped off the image of a vertical arc in $X \times\left[t_{i-1}, t_{i}\right]$. Thus we can easily make sure that $f_{i}^{\prime}$ still satisfies

$$
f_{i}^{\prime}\left(X \times\left[t_{q-1}, t_{q}\right]\right) \subset N_{\delta_{i}}\left(h\left(D_{j}^{k}\left[t_{q-1}, t_{q}\right]\right)\right) \subset N_{\gamma_{i-1}}\left(h\left(D_{j}^{k}\left[t_{q-1}, t_{q}\right]\right)\right) .
$$

Further, the choice of $\delta_{i}$ ensures that $f_{i}^{\prime}(\{x\} \times I) \subset N_{\gamma_{i-1}}\left(F_{j}(r(x))\right)$. Hence we see that $f_{i}^{\prime}$ satisfies the conclusions of the claim with $\delta_{i}$ replaced by $\gamma_{i-1}$. 
The change from $f_{i}^{\prime}$ to $f_{i-1}$ involves a finite number of Type II pipings in $X \times\left[t_{i-2}, t_{i}\right]$. What actually happens is that the image set is moved by an ambient isotopy, then reparametrized, then moved by an ambient isotopy, then reparametrized again, and so forth. The numbers $s_{q}^{i-1}$ and $\eta_{i-1}$ have been chosen in such a way that no point is moved by more than one of those ambient isotopies. (They are supported on disjoint sets.) But the reparametizations leave the images of the fibers $\{x\} \times I$ setwise fixed. Thus property (iv) of the isotopy $H_{t}$ ensures that

$$
f_{i-1}(\{x\} \times I) \subset N_{\eta_{i-1}}\left(F_{j}(r(x))\right) \subset N_{\delta_{i-1}}\left(F_{j}(r(x))\right)
$$

for every $x \in X$.

Also, the complicated construction of the Type II piping was specifically designed to avoid moving any part of the image of $X \times\left[t_{q-1}, t_{q}\right]$ out of $N_{\delta_{t-1}}\left(h\left(D_{j}^{k}\left[t_{q-1}, t_{q}\right]\right)\right)$. If a point was ever in danger of being pulled out of its correct neighborhood by one of the isotopies, we first moved it out of the way. Therefore $f_{i-1}\left(X \times\left[t_{q-1}, t_{q}\right]\right) \subset N_{\delta_{i-1}}\left(h\left(D_{j}^{k}\left[t_{q-1}, t_{q}\right]\right)\right)$ for each $q$. This completes the proof of the claim.

The constructions above have now produced a map $f_{1}$. We finally obtain the map $f$ that we are looking for by doing all the Type I pipings in $X \times\left[t_{0}, t_{1}\right]$ to $f_{1}$. The choice of $\delta_{1}$ then guarantees that $f$ satisfies $f(\{x\} \times I) \subset N_{\delta_{0}}\left(F_{j}(r(x))\right) \subset N_{\varepsilon / 3}\left(F_{j}(r(x))\right)$ for each $x \in X$ and $f\left(X \times\left[t_{q-1}, t_{q}\right]\right) \subset N_{\delta_{0}}\left(h\left(D_{j}^{k}\left[t_{q-1}, t_{q}\right]\right)\right) \subset N_{\varepsilon / 3}\left(h\left(D_{j}^{k}\left[t_{q-1}, t_{q}\right]\right)\right)$ for each $q$.

We now complete the proof of the Lemma by checking that $f$ satisfies all of the conclusion.

(1) We never change $f_{i} \mid X \times\{0\}$, so $f(x, 0)=f_{n}(x, 0)=x$ for every $x \in X$.

(2) As noted above, $f(x, t) \in N_{\varepsilon / 3}\left(F_{j}(r(x))\right)$ for every $(x, t) \in X \times I$. The choice of $\alpha$ together with the fact that $f(x, t) \in N_{\varepsilon / 3}\left(h\left(D_{j}^{k}\left[t_{q-1}, t_{q}\right]\right)\right)$ whenever $t_{q-1} \leq t \leq t_{q}$ shows that $f(x, t) \in N_{\varepsilon}\left(h\left(D_{j}^{k}[t]\right)\right)$.

(3) Since all the modifications to $f_{n}$ were done in the complement of $f_{n}\left(J_{0}\right)$, we still have $f_{n}\left(J_{0}\right)=f\left(J_{0}\right) \subset f(X \times I)$ and $\operatorname{dim} J_{0} \leq k-2$.

(4) Consider $S_{0}=S(f)$. Then $S_{0}^{(k-3)} \supset S^{(k-3)}$. The difference is that each $(k-2)$ simplex $\sigma \subset S$ has been replaced by an annulus $\Sigma \approx(\partial \sigma) \times I$ with $\Sigma \subset(\pi \sigma) \times I$ (refer to Figure 5). Notice that for each $i$ and each $\sigma$,

$$
\begin{aligned}
(\pi \sigma) \times\left[t_{i-1}, t_{i}\right] & \searrow(\pi \sigma) \times\left\{t_{i}\right\} \cup(\pi \partial \sigma) \times\left[t_{i-1}, t_{i}\right] \\
& \cup\left(\Sigma \cap X \times\left[t_{i-1}, t_{i}\right]\right) \\
& \searrow(\pi \sigma) \times\left\{t_{i}\right\} \cup(\pi \partial \sigma) \times\left[t_{i-1}, t_{i}\right]
\end{aligned}
$$


Now $f \mid \Sigma$ is an embedding and each such $\Sigma$ is matched with another $\Sigma^{\prime} \subset S_{0}$ just like it such that $f(\Sigma)=f\left(\Sigma^{\prime}\right)$. Also, the only place where $\Sigma$ attaches to $S^{(k-3)}$ is in $\partial \sigma$. Therefore there is a collapse $\xi: f(X \times I) \searrow$ $f(X \times\{1\}) \cup f\left(J_{0}\right)$. Further, we can time $\xi$ so that any simplex in $X \times\left[t_{i-1}, t_{i}\right]$ which is collapsed by $\xi$ is collapsed during the time interval $t_{i-1} \leq t \leq t_{i}$. If $B$ is any simplex of $X$ then $\xi_{t}(f(B \times I)) \subset f(B \times I)$ for all $t$. We claim that $\xi$ is a $\left(j, \varepsilon, f\left(J_{0}\right)\right)$-collapse. The argument is the same as that given at the beginning of the proof of Lemma 1 .

3. Building $(j, \varepsilon, \varnothing)$-collapses. In this section we will continue the construction begun in $\S 2$. By attaching certain polyhedra to $f(X \times I)$ along $f\left(J_{0}\right)$, we will engulf the $(k-1)$-dimensional polyhedron $X$ in a $k$-dimensional polyhedron for which there is a $(j, \varepsilon, \varnothing)$-collapse. We first state a definition. It should be noted that this definition is one of those which differs slightly from the corresponding one in [2].

Definition. Let $A$ be a polyhedron and $\varepsilon>0$. A homotopy $g$ : $A \times I \rightarrow R^{k+2}$ is a $(j, \varepsilon)$-homotopy if

(a) $g_{t}(A) \subset N_{\varepsilon}\left(h\left(D_{j}^{k}[t, 1]\right)\right)$ for each $t$,

(b) $g_{t}\left|g_{0}^{-1}\left(N_{\varepsilon}\left(h\left(D_{j}^{k}[t, 1]\right)\right)\right)=g_{0}\right| g_{0}^{-1}\left(N_{\varepsilon}\left(h\left(D_{j}^{k}[t, 1]\right)\right)\right)$ for each $t$, and

(c) $g_{t}(x) \in N_{\varepsilon}\left(F_{j}\left(r\left(g_{0}(x)\right)\right)\right)$ for every $x \in A$ and $t \in I$.

To begin the construction, let $X, f$, and $J_{0}$ be as in Lemma 1. Define $Y$ to be the abstract union

$$
Y=f(X \times I) \cup_{f\left(J_{0}\right)} f\left(J_{0}\right) \times I
$$

where each $x \in f\left(J_{0}\right) \subset f(X \times I)$ is identified with $(x, 0) \in f\left(J_{0}\right) \times I$. Let $g: Y \rightarrow R^{k+2}$ be the map defined by

$$
g(y)= \begin{cases}y & \text { if } y \in f(X \times I), \\ \psi^{j}(z, s) & \text { if } y=(z, s) \in f\left(J_{0}\right) \times I .\end{cases}
$$

LEMMA 2. For every $\varepsilon^{\prime}>0$ there is a $\delta^{\prime}>0$ such that if $X \subset$ $N_{\delta^{\prime}}\left(h\left(D_{j}^{k}[0]\right)\right)$ is a compact $(k-1)$-dimensional polyhedron and $Y$ is constructed as in the paragraph above, then there is a collapse $\lambda: Y \searrow C$ with $g \circ \lambda_{t} a\left(j, \varepsilon^{\prime}\right)$-homotopy.

Proof. This is just a formal statement of a result which is proved on $\mathrm{p}$. 410 of [2] and which is also stated as Lemma 4.1 of [3]. The collapse consists of two parts. Use $\xi$ (the collapse defined in Lemma 1) to collapse $f(X \times I)$ to $f\left(J_{0}\right) \cup f(X \times\{1\})$ and at the same time, as bits of $f\left(J_{0}\right)$ are exposed, start to collapse them down the product structure of $f\left(J_{0}\right) \times$ $I$-always keeping the second part of the collapse a little behind $\xi$. 
More precisely, $\lambda$ is constructed as follows. Choose a small positive number $\beta$. First collapse $f(X \times I)$ to $f(X \times[\beta, 1]) \cup f\left(J_{0} \cap X \times[0, \beta]\right)$ using $\xi$. Then collapse $f\left(J_{0}\right) \times I$ to

$$
\left(f\left(J_{0} \cap X \times[\beta, 1]\right) \times I\right) \cup\left(f\left(J_{0} \cap X \times[0, \beta]\right) \times[\beta, 1]\right)
$$

by collapsing straight down the product structure. Next continue the collapse of $f(X \times I)$ to $f(X \times[2 \beta, 1]) \cup f\left(J_{0}\right)$ and follow that by a further collapse down the product structure of $f\left(J_{0}\right) \times I$ to

$$
\left(f\left(J_{0} \cap X \times[2 \beta, 1]\right) \times I\right) \cup\left(f\left(J_{0} \cap X \times[0,2 \beta]\right) \times[2 \beta, 1]\right) .
$$

Continue to alternate back and forth in that manner.

If $\delta^{\prime}$ is small enough, $\psi^{j} \mid f\left(J_{0}\right)$ will have the property that $\psi^{j}(y, t)$ $\in N_{\varepsilon^{\prime}}\left(h\left(D_{j}^{k}[t, 1]\right)\right)$ for each $(y, t) \in f\left(J_{0}\right) \times I$. Therefore $g \circ \lambda_{t}$ will be a $\left(j, \varepsilon^{\prime}\right)$-homotopy.

Our next job will be to work on the singular set of $g$ and do some piping to modify it. Before doing so, however, we must change the way in which $g(Y)$ is parametrized. The problem is that for $(z, t) \in f\left(J_{0}\right) \times I$, we cannot be certain that $g(z, t) \in N_{\varepsilon^{\prime}}\left(h\left(D_{j}^{k}[t]\right)\right)$, but only that $g(z, t) \in$ $N_{\varepsilon^{\prime}}\left(h\left(D_{j}^{k}[t, 1]\right)\right)$.

Suppose $\beta$ in the proof above is of the form $\beta=1 / m$ where $m$ is an integer. Define $F \subset f\left(J_{0}\right) \times I$ by

$$
F=\bigcup_{i=1}^{m}\left(f\left(J_{0} \cap X \times[0, i / m]\right)\right) \times[(i-1) / m, i / m] .
$$

Notice that the image of any vertical segment from $f\left(J_{0}\right) \times I-F$ has small diameter and that if $(z, t) \in F$ then $g(z, t) \in N_{\varepsilon^{\prime}}\left(h\left(D_{j}^{k}[t]\right)\right)$. Let $F_{0}$ be a small neighborhood of $F$ in $f\left(J_{0}\right) \times I$ with the property that there is a PL homeomorphism $H: F_{0} \rightarrow f\left(J_{0}\right) \times I$. The homeomorphism $H$ should be chosen to have two further properties: $H$ preserves first coordinates, and $\operatorname{supp}(H)$ is contained in a small neighborhood of the frontier of $F_{0}$ in $f\left(J_{0}\right) \times I$.

Now define $Y^{*}=f(X \times I) \cup F_{0}$ where each $x \in f\left(J_{0}\right)$ is identified with $H^{-1}(x, 0) \in F_{0}$ and define $g^{*}: Y^{*} \rightarrow R^{k+2}$ by

$$
g^{*}(x)= \begin{cases}x & \text { if } x \in f(X \times I), \quad \text { and } \\ g(H(x)) & \text { if } x \in F_{0} .\end{cases}
$$

If $H$ is chosen carefully enough, $g^{*}$ will have the property that for every $(z, t) \in F_{0}, g^{*}(z, t) \in N_{\varepsilon^{\prime}}\left(F_{j}(r(z))\right) \cap N_{\varepsilon^{\prime}}\left(h\left(D_{j}^{k}[t]\right)\right)$. 
Put $g^{*}$ in general position, keeping $f(X \times I)$ fixed, and let $S=$ $S\left(g^{*}\right)$. Since $g^{*} \mid f(X \times I)$ is an embedding,

$$
\begin{aligned}
\operatorname{dim} S & \leq \operatorname{dim} f(X \times I)+\operatorname{dim} F_{0}-(k+2) \\
& \leq k+(k-1)-(k+2)=k-3 .
\end{aligned}
$$

Define $J_{1}$ to be $\operatorname{Trail}_{\lambda}\left(S^{(k-4)}\right)$. Then $\operatorname{dim} J_{1} \leq k-3$. As in the proof of Lemma 1 , we wish to use a piping argument to punch a hole in each top-dimensional simplex of $S$ so that there is a $\left(j, \varepsilon, g^{*}\left(J_{1}\right)\right)$-collapse of $g^{*}\left(Y^{*}\right)$. That is the first step in an inductive argument which will eventually produce a $(j, \varepsilon, \varnothing)$-collapse.

Lemma 3. (Second Piping Lemma.) For every $\varepsilon>0$ there is a $\delta>0$ such that if $X \subset N_{\delta}\left(h\left(D_{j}^{k}[0]\right)\right)$ is a compact $(k-1)$-dimensional polyhedron, then $f, Y^{*}, g^{*}, \lambda$, and $J_{1}$ can be constructed in such a way that $f$ is homotopic rel $X \times\{0,1\} \cup f^{-1}\left(J_{1}\right)$ to a $P L$ map $\bar{f}: X \times I \rightarrow R^{k+2}$ and $g^{*} \mid F_{0}$ is homotopic rel $H^{-1}\left(J_{0} \times\{0\}\right) \cup J_{1}$ to a $P L$ map $\bar{g}: F_{0} \rightarrow R^{k+2}$ such that there is a $\left(j, \varepsilon, \bar{g}\left(J_{1}\right)\right)$-collapse $\theta: \bar{f}(X \times I) \cup \bar{g}\left(F_{0}\right) \searrow \bar{g}\left(J_{1}\right) \cup$ $C_{1}$.

Proof. Let $\alpha, \beta>0$ and $0=t_{0}<t_{1}<\cdots<t_{n}=1$ be exactly as at the beginning of the proof of Lemma 1 . Choose $\delta$ to be the $\delta^{\prime}$ of Lemma 2 corresponding to

$$
\boldsymbol{\varepsilon}^{\prime}=\min _{i=1, \ldots, n}\left\{\operatorname{dist}\left(h\left(D_{j}^{k}\left[0, t_{i-1}\right]\right), h\left(D_{j}^{k}\left[t_{i}, 1\right]\right)\right)\right\} .
$$

Now suppose $X \subset N_{\delta}\left(h\left(D_{j}^{k}[0]\right)\right)$ and that $f, Y^{*}, g^{*}, \lambda, S$, and $J_{1}$ are all defined as above. Put $g^{*}$ in general position.

As usual, the fact that $g^{*}$ is in general position implies that the top-dimensional simplices of $S$ come in pairs, each of which is identified under $g^{*}$. Let $\left\{\sigma_{1}, \sigma_{2}\right\}$ be a matched pair of $(k-3)$-simplices in $S$ with $\sigma_{1} \subset f(X \times I)$ and $\sigma_{2} \subset F_{0}$. The situation is not symmetric as it was in the proof of Lemma 1, so there are now three cases to consider.

Case 1. $\sigma_{1} \subset f\left(X \times\left[t_{i-1}, t_{i}\right]\right)$ and $\sigma_{2} \subset f\left(J_{0}\right) \times\left[t_{i-1}, t_{i}\right]$ for some $i$.

Case 2. $\sigma_{1} \subset f\left(X \times\left[t_{i}, t_{i+1}\right]\right)$ and $\sigma_{2} \subset f\left(J_{0}\right) \times\left[t_{i-1}, t_{i}\right]$ for some $i$.

Case 3. $\sigma_{1} \subset f\left(X \times\left[t_{i-1}, t_{i}\right]\right)$ and $\sigma_{2} \subset f\left(J_{0}\right) \times\left[t_{i}, t_{i+1}\right]$ for some $i$. One of these three cases must occur by the choice of $\varepsilon^{\prime}$. 


\section{Construction of one pipe.}

Suppose $\left\{\sigma_{1}, \sigma_{2}\right\}$ is a pair which fits either Case 1 or Case 2. (Pairs which fit Case 3 can present a special difficulty. Later in the proof we will use a small trick to change each Case 3 pair into a Case 1 pair and thus avoid that problem.) We will pipe the singularity over the edge of $g^{*}\left(Y^{*}\right)$ in two steps. Step 1 will push it off the fin (but into $f(X \times I)$ ) and is just like the construction of one pipe in Lemma 1. Step 2 is then used to push things off $f(X \times I)$ and involves some new complications.

If Case 1 is the case which occurs initially, do a Type I piping by pushing $g^{*}\left(\sigma_{1}\right)$ along the image of a vertical arc in $F_{0}$ above the barycenter of $\sigma_{2}$. The result is a new pair which fits Case 2.

If Case 2 is the one which occurs, we do a Type II piping. This time we push $g^{*}\left(\sigma_{2}\right)$ along the image of an arc above the barycenter of $\sigma_{1}$. By general position there will be no points of $S(f)$ above the barycenter of $\sigma_{1}$ and so we can do this without obstruction. The result of this piping is a new pair which fits Case 1 again.

By alternating back and forth between Type I and Type II moves, we will eventually reach the top edge of $F_{0}$. Since $F_{0}$ consists of only part of $f\left(J_{0}\right) \times I$, we are likely to push the singularity off the edge of the fin before we reach the 0 -level. This completes Step 1.

REMARK. Notice that we were forced by the lack of symmetry to do things in a very particular way. We make certain that we do the Type I pipings in such a way that Case 1 changes to Case 2. If we were to do the Type I pipings in the other obvious way, then a pair which fits Case 3 would result and we would be stuck. The reason is simply that an arc above $\sigma_{2}$ might not reach all the way to the $t_{i-1}$-level of $f\left(J_{0}\right) \times I$.

Assume that Step 1 has been completed. The result is a hole punched in that part of the intersection between $F_{0}$ and $f(X \times I)$ represented by $\left\{\sigma_{1}, \sigma_{2}\right\}$. Unfortunately the top of the fin is attached to $f(X \times I)$, so we pay for this improvement in the form of a new self-intersection in $f(X \times I)$. Figure 6 shows a picture of what has happened so far in the domain.

As stated above, the way in which we plan to deal with the new singularities is by continuing to pipe, but now doing so along two sheets of $f(X \times I)$. So we focus our attention on exactly what the preimages of the singularities look like all the way back in $X \times I$.

Let $a$ be the point of $f\left(J_{0}\right)$ directly above the barycenter of $\sigma_{2}$ and let $\sigma_{1}^{*}$ denote the preimage of the adjusted $\sigma_{1}$ under $f$. Notice that $\sigma_{1}^{*}$ will consist of an annulus $\left(S^{k-4} \times[0,1]\right)$ together with a $(k-2)$-complex 


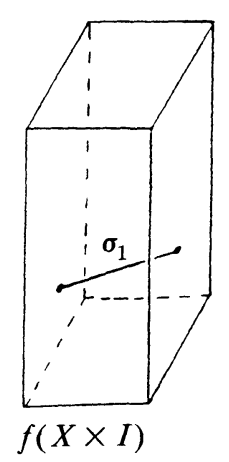

Before

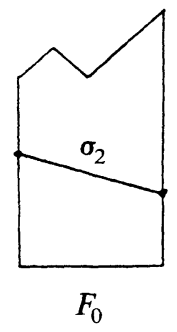

Figure 6

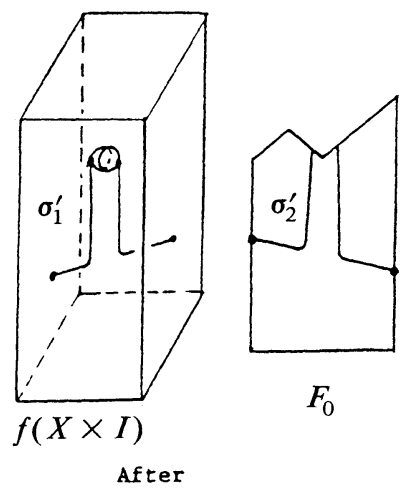

attached to that annulus. If $\pi\left(f^{-1}(a)\right)$ is in the interior of a $(k-1)$ simplex and if we do Step 1 carefully, then that $(k-2)$-complex will be exactly a $(k-2)$-sphere attached to the free boundary component of the annulus as pictured in Figure 7 . In general, the $(k-2)$-complex will be the union of a finite number of $(k-2)$-cells, one for each $(k-1)$-simplex of $X$ which contains $\pi\left(f^{-1}(a)\right)$. These cells are joined together along their common boundary. Let $C$ denote their union. Now $f(C) \cap f\left(J_{0}\right)$ will consist exactly of a $(k-4)$-sphere, $A$, which contains $a$ in its interior relative to $f\left(J_{0}\right)$. We can write $C$ as the join of $f^{-1}(A)$ and a 1-complex $K^{1}$. Let $B^{2}$ denote the cone on $K^{1}$. We can assume that $B^{2}$ is contained in a small neighborhood of $\sigma_{1}^{*}$ as indicated in Figure 7 and that $B^{2} \cap \sigma_{1}^{*}$ $=K^{1}$.

Step 2 now consists of pushing $B^{2}$ straight along $f(X \times I)$ towards $f(X \times\{0\})$. This is done by means of the same back-and-forth alternation between Type I and Type II pipings as always, but there are some new complications.

Whereas earlier we pushed only the barycenter of a simplex, we now push the entire image of $B$ up, keeping $f(A)$ fixed. We use Type I pipings to push $f(B)$ up along $f\left(J_{0}\right)$. This works just like the Type I pipings in the proof of Lemma 1. But the Type II pipings are different. We take an arc $\alpha$ above $B$, push its image up near the $t_{t}$-level, and then reparametrize in such a way that not only is the arc incorporated into the slice $X \times\left[t_{l-1}, t_{l}\right]$, but so is all of $B$. Figure 8 shows the result in $X \times I$.

There is now another obstacle to be faced. As we push $f(B)$ up along $f\left(J_{0}\right)$, everything is simply a product until we reach the top of $f\left(J_{0}\right)$. There we will see part of $f(S(f))$. Attached to this part of $f(S(f))$ may be $(k-2)$-dimensional annuli of $f(S(f))$ as shown in Figure 7. We must avoid pushing $f\left(K^{1}\right)$ into those annuli because that would create still 

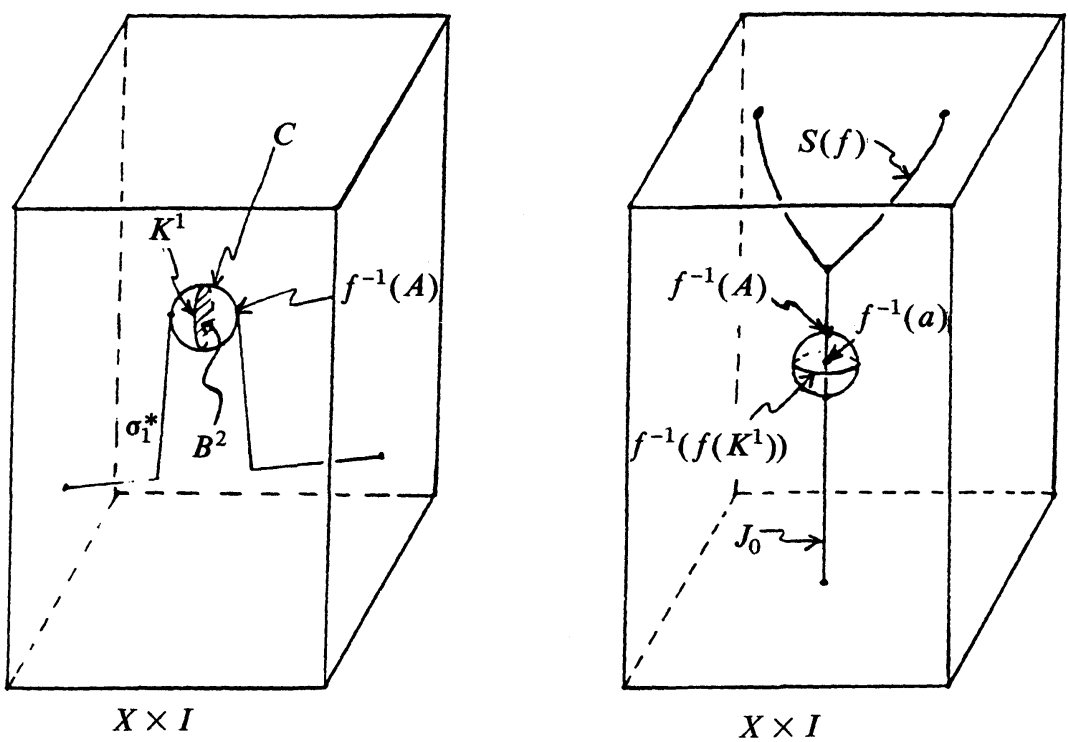

FIGURE 7
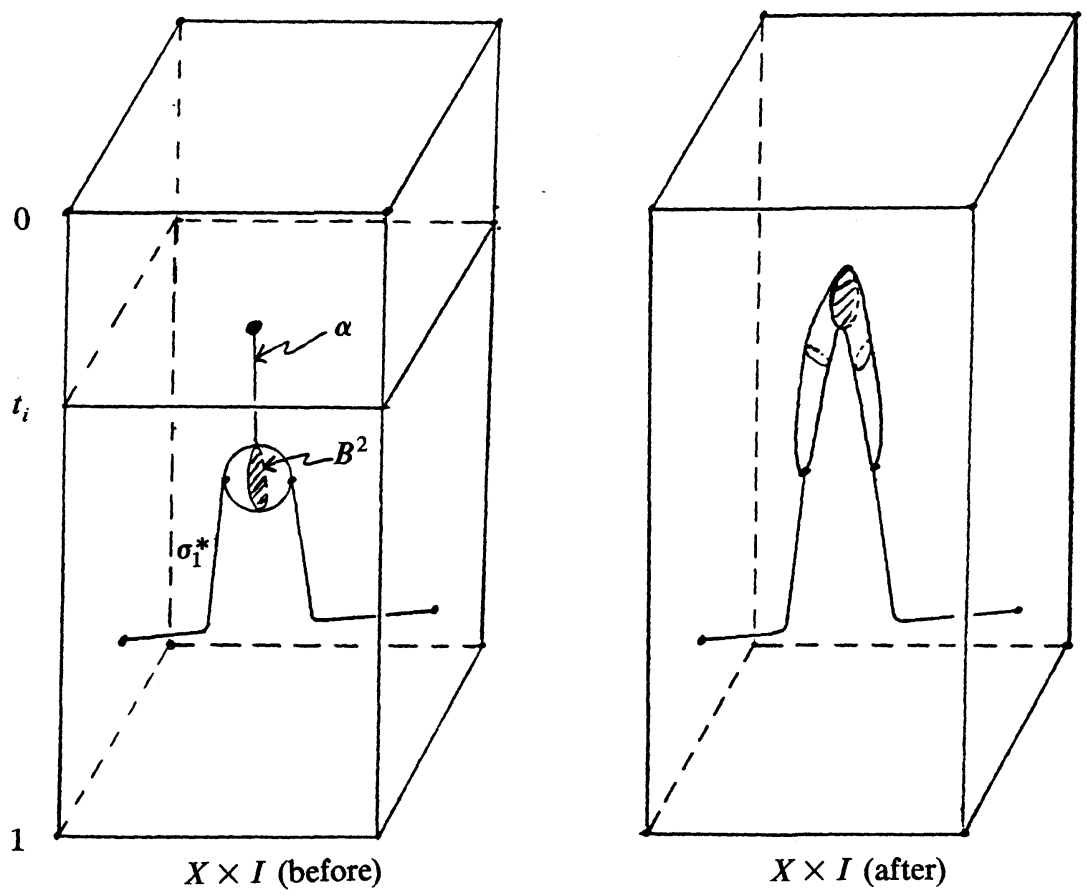

FIGURE 8 


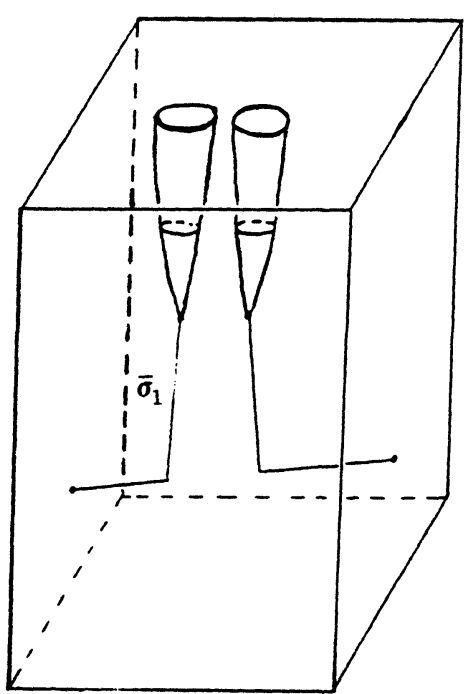

$X \times I$

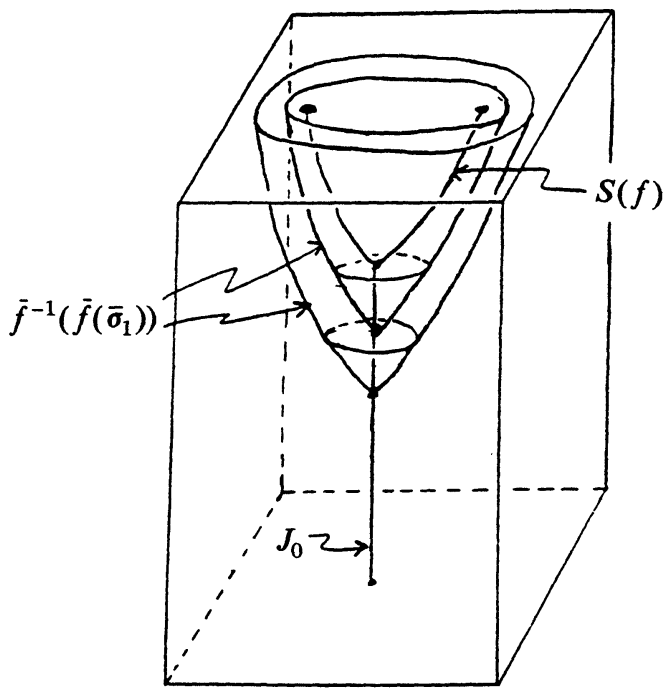

$X \times I$

FigURE 9

more self-intersections in $f(X \times I)$. (Other sheets of $f(X \times I)$ are attached there.) But $\left(\pi\left(K^{1}\right) \times I\right) \cap S(f)$ will consist of a finite set of points, one for each $(k-2)$-annulus of $f(S(f))$ attached to the $(k-3)$ simplex above $a$. To avoid pushing $K^{1}$ into $f(S(f))$, simply push that point out radially and then continue up through the hole in $f(S(f))$ which was created in the proof of Lemma 1.

Figure 9 shows the results of these changes in the domain, $X \times I$. Notice that $\sigma_{1}^{*}$ has been replaced by a complex $\bar{\sigma}_{1}$ which collapses and that part of $\bar{\sigma}_{1}$ is identified, under $\bar{g} f$, with the sets labeled $\bar{f}^{-1}\left(\bar{f}\left(\bar{\sigma}_{1}\right)\right)$ in the right-hand panel. It is not possible to draw a dimensionally accurate picture of what occurs in the range, but Figure 10 gives the general idea.

There is one last problem to be faced. In Step 2 we push $f\left(B^{2}\right)$ near to parts of $f(S(f))$. But there are other sheets of $f(X \times I)$ (not shown in the figures above) which cross there. Thus we have probably created new intersections with those sheets of $f(X \times I)$. The way in which we intend to avoid this problem is simple: the singularities we are worrying about in Step 2 are ones which were created (by us) in the course of doing Step 1 and so we will just go back and do Steps 1 and 2 in such a way that the problem does not arise.

Consider what happened in Step 1 when $\sigma_{1}$ was pushed off the edge of the fin. Instead of pushing $\sigma_{1}$ all the way through $f(X \times I)$, we could just push it into $f(X \times I)$ and stop there. Then, at the end of Step 1, the 

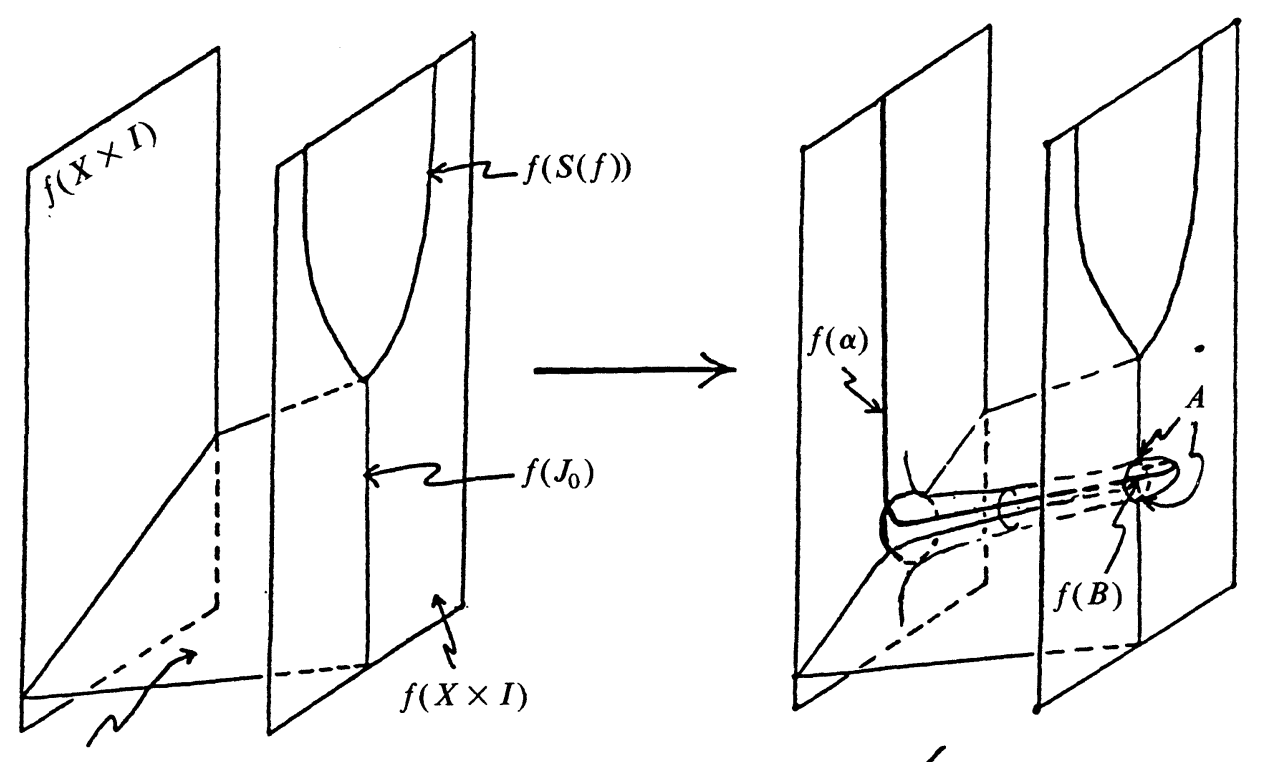

$g^{*}\left(F_{0}\right)$
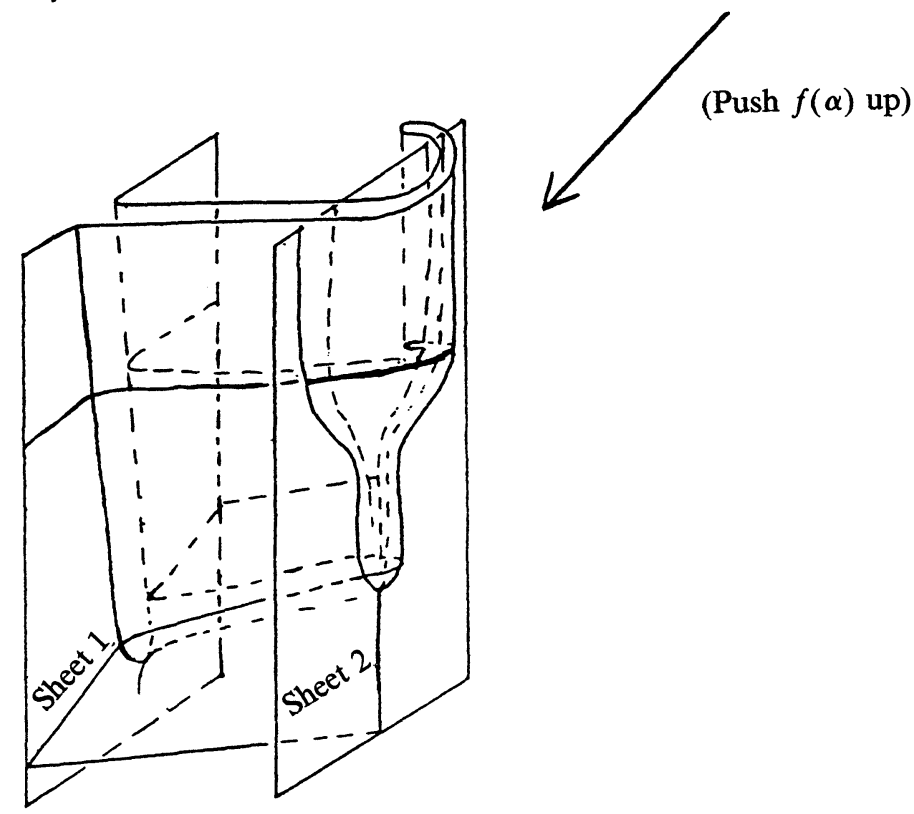

Figure 10

fin would look the same as before but the two sheets of $f(X \times I)$ would have a $k$-dimensional set in common. This $k$-dimensional set is the join of $B$ and $f^{-1}(A)$. If we now proceed to do Step 2, the construction will be the same except that we will end up with the two sheets still intersecting in a $k$-dimensional set. We push $B^{2}$ off the top of $f(X \times I)$, so now the intersection of the two sheets is homeomorphic with the join of $B^{2}$ and 
$f^{-1}(A)$ minus a neighborhood of $B^{2}$, which collapses via a collapse which induces a $(j, \varepsilon)$-homotopy.

This means that when we are finished with Step 2, the singular set of $\bar{f}$ will be $k$-dimensional. The picture will be a little different from that presented first. In Figure 10, the part of Sheet 1 which pokes through Sheet 2 in the second and third pictures is projected to the left so that it lies flat in Sheet 2.

\section{Construction of all the pipes simultaneously.}

So far we have explained how an individual pipe is constructed. In order to complete the proof of the Lemma, we must deal with two remaining points: first, how to avoid pairs which fit Case 3, and second, how to do all this and maintain the desired $\varepsilon$-control so that the collapse $\theta$ exists.

Here is a trick which will exchange each pair which fits Case 3 for one which fits Case 1. Given the partition $0=t_{0}<t_{1}<\cdots<t_{n}=1$, choose a refinement $0=t_{0}^{\prime}<t_{0}^{\prime}<\cdots<t_{\mathrm{m}}^{\prime}=1$ of much smaller mesh. Now suppose that $\delta$ was chosen to be small compared with the mesh of the new partition. Then each pair of $(k-3)$-simplices in $S$ would fit one of the three cases listed above, but this time relative to the finer partition. Let $\left\{\sigma_{1}, \sigma_{2}\right\}$ be a pair such that $\sigma_{1} \subset f\left(X \times\left[t_{i-1}^{\prime}, t_{i}^{\prime}\right]\right)$ and $\sigma_{2} \subset f\left(J_{0}\right) \times$ $\left[t_{t}^{\prime}, t_{i+1}^{\prime}\right]$. Simply reparametrize the map $f$ so that the barycenter of $\sigma_{1}$ lies in $f\left(X \times\left[t_{1}^{\prime}, t_{i+1}^{\prime}\right]\right)$. This changes the pointwise control on $g^{*}$ by very little and does not cause trouble since we do this one time only. Now each pair fits either Case 1 or Case 2 relative to the first partition.

The collapse $\theta$ is constructed from the composition of four different collapses.

$$
\begin{gathered}
\bar{f}(X \times I) \searrow f(X \times\{1\}) \cup f\left(J_{0}\right) \cup J_{1} \cup \bar{f}(S(\bar{f})) \cup\left[\bar{f}(X \times I) \cap \bar{g}\left(F_{0}\right)\right] \\
\searrow f(X \times\{1\}) \cup f\left(J_{0}\right) \cup J_{1} \cup\left[\bar{f}(X \times I) \cap \bar{g}\left(F_{0}\right)\right] .
\end{gathered}
$$

But

$$
J_{1} \cup f\left(J_{0}\right) \cup \bar{g}\left(F_{0}\right) \searrow \bar{f}(S(\bar{g})) \cup J_{1} \cup g\left(J_{0} \times\{1\}\right) \searrow J_{1} \cup g\left(J_{0} \times\{1\}\right) \text {. }
$$

The way in which $\theta$ is constructed from those four collapses is just like the construction of $\lambda$ in the proof of Lemma 2. Achieving the desired $\varepsilon$-control of course requires a much more careful choice of $\delta$. The details are just like those in the proof of Lemma 1 and so we omit them.

This completes the proof of Lemma 3.

We now come to the large inductive construction which is the culmination of Sections 2 and 3. 
LeMMA 4. For every $\varepsilon>0$ there exists $\delta>0$ such that if $X$ is a compact, $(k-1)$-dimensional polyhedron in $N_{\delta}\left(h\left(D_{j}^{k}[0]\right)\right)$, then $X \subset Y \subset$ $N_{\varepsilon}\left(h\left(D^{k}\right)\right)$ where $Y$ is a $k$-dimensional polyhedron for which there is a $(j, \varepsilon, \varnothing)$-collapse $\xi: Y \searrow C$.

Proof. In Lemma 1 we showed that $\delta$ could be chosen so that $X$ is contained in a polyhedron $f(X \times I)$ for which there is a $\left(j, \varepsilon, J_{0}\right)$-collapse with $\operatorname{dim} J_{0} \leq k-2$. Then in Lemma 3 we showed that $\delta$ could be further refined and $f(X \times I)$ modified and enlarged to a polyhedron for which there is a $\left(j, \varepsilon, J_{1}\right)$-collapse with $\operatorname{dim} J_{1} \leq k-3$. These are obviously the first two steps of an inductive proof of Lemma 4; we keep reducing the dimension of the image of the collapse until eventually it is empty. Rather than get bogged down in the notation of a full inductive proof of the lemma, we will concentrate instead on just the third step and then the inductive construction will be clear.

The construction described below is built on that of Lemma 3. We claim that for any given $\varepsilon$ there is an $\varepsilon^{\prime}>0$ such that if the construction of Lemma 3 is done to within a tolerance $\varepsilon^{\prime}$, then we can do the construction below so carefully that when we are done we have a $\left(j, \varepsilon, J_{2}\right)$-collapse with $\operatorname{dim} J_{2} \leq k-4$. Proving the claim requires an understanding of the construction below and then the details are just like those in the choice of $\delta$ in Lemma 1, so we omit them.

Choose $\delta^{\prime}$ to be the $\delta^{\prime}$ of Lemma 3 corresponding to the $\varepsilon^{\prime}$ of the claim above. Suppose $X \subset N_{\delta^{\prime}}\left(h\left(D_{j}^{k}[0]\right)\right)$ is a compact $(k-1)$ dimensional polyhedron. Let $f_{0}: X \times I \rightarrow R^{k+2}$ be the map given by Lemma 1. There exists $J_{0} \subset f_{0}(X \times I)$ and a $\left(j, \varepsilon^{\prime}, J_{0}\right)$-collapse of $f_{0}(X \times I)$ to $J_{0} \cup f_{0}(X \times\{1\})$. Now let $F_{0}$ be the fin constructed in Lemma 3, let $Y_{1}=X \times I \cup F_{0}$ and let $f_{1}: Y_{1} \rightarrow R^{k+2}$ be the PL map of Lemma 3. There is a $(k-3)$-dimensional polyhedron $J_{1} \subset f_{1}\left(Y_{1}\right)$ and a $\left(j, \varepsilon^{\prime}, J_{1}\right)$-collapse $\theta: f_{1}\left(Y_{1}\right) \searrow J_{1} \cup C_{1}$.

Define $Y_{2}^{\prime}$ to be $f_{1}\left(Y_{1}\right) \cup\left(J_{1} \times I\right)$ where $x \in J_{1} \subset f_{1}\left(Y_{1}\right)$ is identified with $(x, 0) \in J_{1} \times I$. Use $\psi^{j}$ to extend the identity on $f_{1}\left(Y_{1}\right)$ to a map $f_{1}^{\prime}$ : $Y_{2}^{\prime} \rightarrow R^{k+2}$. As in the proof of Lemma 3 , there exists a subpolyhedorn $F_{1}$ of $J_{1} \times I$ and a PL homeomorphism $H: F_{1} \rightarrow J_{1} \times I$ which preserves first coordinates and has the following property: if $(z, t) \in F_{1}$, then $f_{2}^{\prime}(H(z, t)) \in N_{\varepsilon^{\prime}}\left(h\left(D_{j}^{k}[t]\right)\right) \cap N_{\varepsilon^{\prime}}\left(F_{j}(r(z))\right)$. Define $Y_{2}$ to be $f_{1}\left(Y_{1}\right) \cup F_{1}$ where $x \in J_{1} \subset f_{1}\left(Y_{1}\right)$ is identified with $H^{-1}(x, 0) \in F_{1}$. Define $f_{2}: Y_{2} \rightarrow$ $R^{k+2}$ to be the identity on $f_{1}\left(Y_{1}\right)$ and $\psi^{j} \circ H^{-1}$ on $F_{1}$. Then $f_{1}(z, t) \in$ $N_{\varepsilon^{\prime}}\left(h\left(D_{j}[t]\right)\right) \cap N_{\varepsilon^{\prime}}\left(F_{j}(r(z))\right)$ for every $(z, t) \in F_{1}$. (We need this kind of pointwise control to make our controlled piping arguments work.) By the 
proof of Lemma 2, there is a collapse $\lambda: Y_{2} \searrow C_{2}$ such that $f_{2} \circ \lambda_{t}$ is a $(j, \varepsilon)$-homotopy.

Shift $f_{2}$ into general position. Since $f_{2} \mid f_{1}\left(Y_{1}\right)$ is an embedding,

$$
\begin{aligned}
\operatorname{dim} S\left(f_{2}\right) & \leq \operatorname{dim} f_{1}\left(Y_{1}\right)+\operatorname{dim} F_{1}-(k+2) \\
& \leq k+(k-2)-(k+2)=k-4 .
\end{aligned}
$$

Define $S$ to be the $(k-5)$-dimensional skeleton of $S\left(f_{2}\right)$ and $J_{2}$ to be $f_{2}\left(\operatorname{Trail}_{\lambda}(S)\right)$. As usual, the proof is completed by homotoping $f_{2}$, rel $J_{2}$, so that $f_{2}\left(Y_{2}\right)$ collapses to $J_{2} \cup f_{2}\left(C_{2}\right)$.

The $(k-4)$-simplices of $S\left(f_{2}\right)$ come in pairs $\left\{\sigma_{1}, \sigma_{2}\right\}$ such that $f_{2}\left(\sigma_{1}\right)=f_{2}\left(\sigma_{2}\right), \sigma_{1} \subset f_{0}(X \times I)$, and $\sigma_{2} \subset F_{1}$. By the same trick as in the proof of Lemma 3, we may assume that there exists an $i$ such that one of the following two cases occurs:

Case 1. $\sigma_{1} \subset f_{0}\left(X \times\left[t_{i-1}, t_{i}\right]\right)$ and $\sigma_{2} \subset J_{1} \times\left[t_{i-1}, t_{i}\right]$,

Case 2. $\sigma_{2} \subset f_{0}\left(X \times\left[t_{i}, t_{i+1}\right]\right)$ and $\sigma_{2} \subset J_{1} \times\left[t_{i-1}, t_{i}\right]$.

For each such pair, we will now construct a pipe. The pipe is constructed in three stages. We first push $f_{2}\left(\sigma_{1}\right)$ off the new fin $f_{2}\left(F_{1}\right)$, then off the original fin $f_{1}\left(F_{0}\right)$, and finally off $f_{0}(X \times I)$. There is only one new aspect to this construction. In the proof of Lemma 3, at the second stage of the construction of one pipe, it was necessary to spread things out to avoid part of $S\left(f_{0}\right)$ above $J_{0}$. We do now want that spreading out to compound on us, so we now argue that, for each pipe, we will have to do that at most once.

Consider $\sigma_{2} \subset F_{1}$. Above the barycenter $b$ of $\sigma_{2}$ there is a point $a \in J_{1}$. Now looking in $Y_{1}$, we find a point $c \in f_{1}\left(S\left(f_{1}\right)\right)$ above $a$. This point $c$ is necessarily a double point of $f_{1}$ by general position. (The set of triple points has codimension two in $S\left(f_{1}\right)$.) The double point $c$ will either be a place where $F_{0}$ crosses itself or a place where $F_{0}$ crosses $f_{0}(X \times I)$. If the latter is the case, we bend the path we pipe along just a little (as shown in Figure 11) so that the pipe goes directly from $F_{1}$ to $f_{0}(X \times I)$. That means that the construction of this particular pipe skips the second stage of the construction and is exactly the same as the construction of one pipe in Lemma 3. Otherwise we push $f_{2}\left(\sigma_{1}\right)$ off $f_{2}\left(F_{1}\right)$ and into $f_{1}\left(F_{0}\right)$, then off $f_{1}\left(F_{0}\right)$ into $f_{0}(X \times I)$ and finally off $f_{0}(X \times I)$. In $F_{0}$, the picture will be as in Figure 12(a) and so no spreading out will be needed in the second stage because there is no part of $S\left(f_{1}\right)$ above $c$. Figure 12(b) is what we have avoided. 


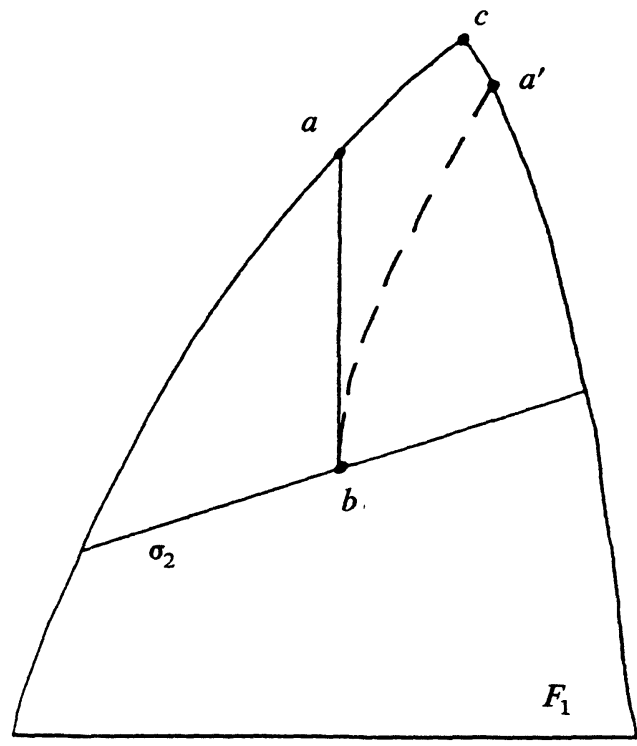

FIGURE 11

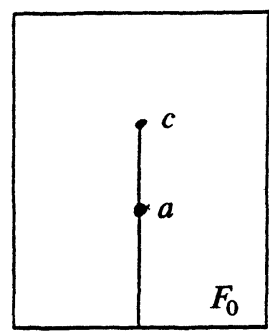

(a)

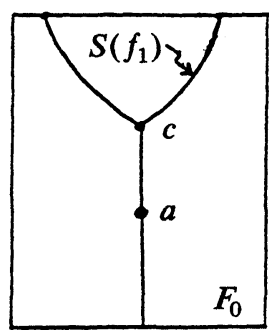

(b)

Figure 12

This completes the proof of Lemma 4.

REMARK. Even in later steps of the inductive proof of Lemma 4, it will only be necessary to do the "spreading out" once. This is because the double points we are concerned with at later stages will always arise as points where the new fin hits $f_{0}(X \times I)$. We always push directly into $f_{0}(X \times I)$.

4. Enlarging $(j, \varepsilon, \varnothing)$-collapses. In this section we show that a $(j, \varepsilon, \varnothing)$-collapse like that built in the preceding section can be enlarged to contain certain specified $(k-1)$-dimensional polyhedra. We first give a name to such a collapse. 
Definition. Suppose $\varepsilon>0$ and $X$ is a compact, $(k-1)$-dimensional polyhedron in $N_{\varepsilon}\left(h\left(D_{j}^{k}[0]\right)\right)$. We say that a collapse $\sigma: Y \searrow C$ is a standard $(j, \varepsilon, \varnothing)$-collapse for $X$ if $Y$ is a $k$-dimensional polyhedron constructed according to the proof of Lemma 4 and $\xi$ is the collapse described there.

The most important features of a standard $(j, \varepsilon, \varnothing)$-collapse are that $Y$ consists of the track of a $(j, \varepsilon)$-homotopy of $X$ with tracks of homotopies of lower dimensional polyhedra attached and that $\xi$ follows those tracks. The next lemma corresponds to Miller's Lemma 9 [2].

Lemma 5. For every $\varepsilon>0$ and for every $\alpha>0$, there exists $\delta>0$ with the following property. If $X \subset N_{\delta}\left(h\left(D_{j}^{k}[0]\right)\right)$ is a compact $(k-1)$ dimensional polyhedron and $\sigma: Y \searrow C$ is a standard $(j, \delta, \varnothing)$-collapse for $X$ and if $Z \subset N_{\delta}\left(h\left(D_{j}^{k}[\alpha]\right)\right)$ is also a compact polyhedron of dimension $\leq k-$ 1 , then there is a $(j, \varepsilon, \varnothing)$-collapse $\xi_{1}: Y_{1} \searrow C_{1}$ such that $Y \cup Z \subset Y_{1}$. Furthermore, $Y_{1} \cap N_{\delta}\left(h\left(D_{j}^{k}[0]\right)\right)=Y \cap N_{\delta}\left(h\left(D_{j}^{k}[0]\right)\right)$.

Proof. Let $r=\operatorname{dim} Z$. By induction it suffices to consider the special case in which $Z \cap Y \supset Z^{(r-1)}$. Again we will just describe the construction of $Y_{1}$ and $\xi_{1}$ and leave the details of the choice of $\delta$ to the reader.

Let $X, Y$ and $\xi: Y \searrow C$ be as in the statement of the Lemma. Then there exists a map $f: X \times I \rightarrow R^{k+2}$ which represents the first stage of the construction of $Y$. Use Lemma 4 to put $Z$ in a standard $(j, \varepsilon, \varnothing)$-collapse $Z^{*}$ with $\theta: Z^{*} \searrow C^{*}$ denoting the actual collapse. Being a standard collapse for $Z, Z^{*}$ contains $f^{*}(Z \times[\alpha, 1])$ for some $(j, \varepsilon)$-homotopy $f^{*}$ of $Z$. When doing the construction of Lemma 4, choose $f^{*}\left(Z^{(r-1)} \times[\alpha, 1]\right)$ to equal $\operatorname{Trail}_{\xi}\left(Z^{(r-1)}\right)$ so that $Y$ and $Z^{*}$ match up nicely. Define $Y^{*}$ to be the abstract union of $Y$ and $Z^{*}$, sewn together along $f^{*}\left(Z^{(r-1)} \times[\alpha, 1]\right)$. Then there is a $(j, \varepsilon, \varnothing)$-collapse $\mu$ : $Y^{*} \searrow C^{*}$ by Lemma 2. Define $g: Y^{*} \rightarrow R^{k+2}$ to be the inclusion on each of the two pieces of $Y^{*}$.

Now put $g$ in general position, keeping $Y$ fixed. Our plan is simply to attach fins to $g\left(Y^{*}\right)$ along $\operatorname{Trail}_{\mu}(S(g))$ and build up a $(j, \varepsilon, \varnothing)$-collapse from $g\left(Y^{*}\right)$. Consider $S(g)$.

$$
\begin{aligned}
\operatorname{dim} S(g) & \leq \operatorname{dim} Y+\operatorname{dim} Z^{*}-(k+2) \\
& \leq k+k-(k+2)=k-2
\end{aligned}
$$

As usual, the top-dimensional simplices of $S(g)$ comes in pairs $\left\{\sigma_{1}, \sigma_{2}\right\}$ where $\sigma_{1} \subset f(X \times I) \subset Y, \sigma_{2} \subset f^{*}(Z \times[\alpha, 1]) \subset Z^{*}$, and $g\left(\sigma_{1}\right)=g\left(\sigma_{2}\right)$. For each pair, construct a pipe (exactly as in the proof of Lemma 1) off the end of $Z^{*}$. Notice that, since $Z^{*}$ lies near $h\left(D_{j}^{k}[\alpha, 1]\right)$, we can do that 
without moving any points of $Y$ near $h\left(D_{j}^{k}[0]\right)$; this is now we achieve the last conclusion of the Lemma.

Let $L_{0}=g\left(\operatorname{Trail}_{\mu}\left(S(g)^{(k-3)}\right)\right)$. After all the pipes have been built, there will be a $\left(j, \varepsilon, L_{0}\right)$-collapse of $g\left(Y^{*}\right)$. As in the proof of Lemma 3, we can attach a fin $F_{0}$ to $g\left(Y^{*}\right)$ along $L_{0}$. Put $F_{0}$ in general position with respect to $g\left(Y^{*}\right)$. Then

$$
\operatorname{dim}\left[F_{0} \cap g\left(Y^{*}\right)\right] \leq(k-1)+k-(k+2)=k-3 .
$$

Once again we construct a pipe for each top-dimensional simplex of $F_{0} \cap g\left(Y^{*}\right)$. But we must be careful how we do so because if we push a simplex of $g\left(Y^{*}\right)$ off $F_{0}$ and into $f(X \times I)$, we will be forced to continue piping all the way along $Y$ to $f(X \times\{0\})$ and will not be able to claim that points near $h\left(D_{j}^{k}[0]\right)$ are left fixed. Just as in the proof of Lemma 4, we have some flexibility about which side of $F_{0}$ we push off. Each double point of $g$ arises from a place where $f^{*}(Z \times[\alpha, 1])$ intersects $f(X \times I)$. Just make sure that when the pipe is pushed off $J_{0}$ it is pushed off into $f^{*}(Z \times[\alpha, 1])$. After these pipes have been built there will be $\left(j, \varepsilon, J_{1}\right)$ collapse of $g\left(Y^{*}\right) \cup F_{0}$, where $\operatorname{dim} J_{1} \leq k-3$.

The next step is to attach a fin $F_{1}$ to $g\left(Y^{*}\right) \cup F_{0}$ along $J_{1}$ and then to pipe top-dimensional simplices of $F_{1} \cap\left(F_{0} \cup g\left(Y^{*}\right)\right)$. This procedure is continued inductively as in Lemma 4 . At later stages of the construction, building one pipe will involve pushing something off a whole sequence of fins. But it is always the case that a fin is attached where a fin which was new at the previous stage intersects $g\left(Y^{*}\right)$, so we can always avoid piping off the edge of the new fin into $f(X \times I)$.

When we apply Lemma 5, we will actually need to do so in a PL submanifold of $R^{k+2}$ which lies near $h\left(D^{k}\right)$, rather than in $R^{k+2}$ itself. The key ingredient which made all the proofs go through was not so much the particular structure of $R^{k+2}$, but rather the existence of the $(j, \varepsilon)$-homotopies we needed. (Recall that we constructed them by using the fact that $h\left(D^{k}\right)$ is an ANR to squeeze things down to $h\left(D^{k}\right)$ and then sliding along the product structure of $D^{k}$.) We could just as well have proved the following Lemma. The only thing which prevented us from doing so immediately was the additional notation which would have been required. The relationship betweens Lemmas 5 and 6 is exactly the same as the relationship between Lemma 9 and Corollary 10 of [2].

LemMa 6. Fix $j<k$. Let $\left\{\Sigma_{\delta} \mid \delta>0\right\}$ denote a set of collections of closed, s-dimensional PL submanifolds of $R^{k+2}$ with each element of $\Sigma_{\delta}$ contained in $N_{\delta}\left(h\left(D^{k}\right)\right)$. Suppose $\left\{\Sigma_{\delta}\right\}$ has the property that for every 
$\varepsilon^{\prime}>0$ there exists $\delta^{\prime}>0$ such that if $S_{1} \in \Sigma_{\delta^{\prime}}$ and $Z$ is a compact subpolyhedron of $S_{1}$ of dimension $\leq s-3$, then there is $S_{2} \in \Sigma_{\varepsilon^{\prime}}$ such that $S_{2}$ contains a $\left(j, \varepsilon^{\prime}\right)$-homotopy of $Z$ and $S_{2} \cap N_{\delta^{\prime}}\left(h\left(D_{j+1}^{k}[0]\right)\right)=S_{1} \cap$ $N_{\delta^{\prime}}\left(h\left(D_{j+1}^{k}[0]\right)\right)$.

Then for every $\varepsilon>0$ and every $\alpha>0$ there exists $\delta>0$ such that if $S \in \Sigma_{\delta}, Y$ is a standard $(j, \delta, \varnothing)$-collapse of dimension $\leq s-2$ in $S$, and $X \subset S \cap N_{\delta}\left(h\left(D_{j}^{k}[\alpha]\right)\right)$ is a compact polyhedron with $\operatorname{dim} X \leq s-3$, then there exists $S^{*} \in \Sigma_{\varepsilon}$ with these properties.

1. $S^{*} \cap N_{\delta}\left(h\left(D_{j+1}^{k}[0]\right)\right)=S \cap N_{\delta}\left(h\left(D_{j+1}^{k}[0]\right)\right)$.

2. $S^{*}$ contains a standard $(j, \varepsilon, \varnothing)$-collapse $Y^{*}$ which in turn contains $Y \cup X$.

3. $\operatorname{dim}\left(Y^{*}-Y\right) \leq \operatorname{dim} X+1$.

4. $Y^{*} \cap N_{\delta}\left(h\left(D_{j}^{k}[0]\right)\right)=Y \cap N_{\delta}\left(h\left(D_{j}^{k}[0]\right)\right)$.

5. $(j, \varepsilon)$-collapses. In this section we relate the $(j, \varepsilon, \varnothing)$-collapses we have been working with to the $(j, \varepsilon)$-collapses of Miller. We show that every $(j, \delta, \varnothing)$-collapse is also a $(j, \varepsilon)$-collapse in the terminology of [2]. Once that has been established, we will really be finished with the proof for Theorem 1, since our Lemma 6 is a codimension two version of Miller's Corollary 10 and Miller's Lemmas 11, 12, and 13 are essentially dimension free-depending only on his Corollary 10. However, for the sake of completeness and for the sake of the reader who is not familiar with [2], we will include a final section in which we sketch the remainder of the proof of Theorem 1 .

Definition [2, pp. 407 and 408]. Let $Y$ and $C$ be subpolyhedra of $N$. A collapse $\sigma: Y \searrow C$ is called a $(j, \varepsilon)$-collapse if $d\left(\xi_{t}(x), \psi_{t}^{j}(x)\right)<\varepsilon$ for every $y \in Y$ and $t \in I$.

LEMMA 7. For every $\varepsilon>0$ and $1 \leq j \leq k$ there exists $\delta>0$ such that each $(j, \delta, \varnothing)$-collapse is also a $(j, \varepsilon)$-collapse.

Proof. Let $\varepsilon>0$ be given. We give the details of the choice of $\delta$. First choose $\gamma>0$ such that $N_{\gamma}\left(F_{j}(y)\right) \cap N_{\gamma}\left(h\left(D_{j}^{k}[t]\right)\right) \subset N_{\varepsilon / 2}\left(F_{j}(y) \cap\right.$ $\left.h\left(D_{j}^{k}[t]\right)\right)$ for every $(y, t) \in h\left(D^{k}\right) \times I$. We next choose a sequence of five $\delta$ 's.

Choose $\delta_{1}>0$ such that if $N_{\delta_{1}}\left(F_{j}\left(y_{1}\right)\right) \cap N_{\delta_{1}}\left(F_{j}\left(y_{2}\right)\right) \neq \phi$ then $N_{\delta_{1}}\left(F_{j}\left(y_{1}\right)\right) \subset N_{\gamma}\left(F_{j}\left(y_{2}\right)\right)$ for every $y_{1}, y_{2} \in h\left(D^{k}\right)$.

Choose $\delta_{2}>0$ such that $N_{\delta_{2}}\left(h\left(D_{j}^{k}[0, t]\right)\right) \cap N_{\delta_{2}}\left(h\left(D_{j}^{k}[t, 1]\right)\right) \subset$ $N_{\gamma}\left(h\left(D_{j}^{k}[t]\right)\right)$ for every $t \in I$. 
Choose $\delta_{3}>0$ such that $\psi_{t}^{j} \mid N_{\delta_{3}}\left(h\left(D_{j}^{k}[t, 1]\right)\right)$ is within $\varepsilon$ of the identity for every $t$.

Choose $\delta_{4}>0$ such that $\psi_{t}^{j}\left(N_{\delta_{4}}\left(h\left(D_{j}^{k}[0, t]\right)\right)\right) \subset N_{\gamma}\left(h\left(D_{j}^{k}[t]\right)\right)$ for every $t \in I$.

Choose $\delta_{5}>0$ such that $d(x, r(x))<\delta_{1}$ for every $x \in N_{\delta_{5}}\left(h\left(D^{k}\right)\right)$.

Define $\delta=\min \left\{\delta_{1}, \delta_{2}, \delta_{3}, \delta_{4}, \delta_{5}\right\}$.

Suppose $\sigma: Y \searrow C$ is a $(j, \delta, \varnothing)$-collapse. We must show that $d\left(\xi_{t}(x), \psi_{t}^{j}(x)\right)<\varepsilon$ for every $(x, t) \in Y \times I$.

Fix $(x, t) \in Y \times I$. Notice that $Y \subset N_{\delta}\left(h\left(D^{k}\right)\right)$, so either $x \in$ $N_{\delta}\left(h\left(D_{j}^{k}[0, t]\right)\right)$ or $x \in N_{\delta}\left(h\left(D_{j}^{k}[t, 1]\right)\right)-N_{\delta}\left(h\left(D_{j}^{k}[0, t]\right)\right)$.

Case 1. $x \in N_{\delta}\left(h\left(D_{j}^{k}[0, t]\right)\right)$. Then $\psi_{t}^{j}(x) \in N_{\gamma}\left(h\left(D_{j}^{k}[t]\right)\right)$ by the choice of $\delta_{4}$ and $\psi_{t}^{j}(x) \in N_{\delta_{1}}\left(F_{j}(r(x))\right)$ by the choice of $\delta_{5}$. Therefore $\psi_{t}^{j}(x) \in N_{\varepsilon / 2}\left(F_{j}(r(x)) \cap h\left(D_{j}^{k}[t]\right)\right)$ by the choice of $\gamma$.

On the other hand, parts (b) and (c) of the definition of $(j, \varepsilon, Z)$-collapse together with the choice of $\delta_{2}$ imply that $\xi_{t}(x) \in N_{\gamma}\left(h\left(D_{j}^{k}[t]\right)\right)$. Part (d) of the definition of $(j, \varepsilon, Z)$-collapse plus the choice of $\delta_{1}$ give $\xi_{t}(x) \in N_{\gamma}\left(F_{j}(r(x))\right)$. The choice of $\gamma$ again gives $\xi_{t}(x) \in N_{\varepsilon / 2}\left(h\left(D_{j}^{k}[t]\right)\right.$ $\left.\cap F_{j}(r(x))\right)$. Since $h\left(D_{j}^{k}[t]\right) \cap F_{j}(r(x))$ is just one point, we have that $d\left(\xi_{t}(x), \psi_{t}^{j}(x)\right)<\varepsilon$ by the triangle inequality.

Case 2. $x \in N_{\delta}\left(h\left(D_{j}^{k}[t, 1]\right)\right)-N_{\delta}\left(h\left(D_{j}^{k}[0, t]\right)\right)$. Then $d\left(x, \psi_{t}^{j}(x)\right)<\varepsilon$ by the choice of $\delta_{3}$ and $\xi_{t}(x)=x$ by part (c) of the definition of $(j, \varepsilon, Z)$-collapse.

6. Proof of Theorem 1. In this section we give a brief outline of the remainder of the proof of Theorem 1. All the ideas are also found on pp. 413-416 of [2]. If $T$ is a triangulation, we use $T^{\prime}$ to denote the first barycentric subdivision of $T$. If $j<k$, we will think of $D^{j}$ as being a subset of $D^{k}$ by identifying $\left(x_{1}, \ldots, x_{j}\right) \in D^{j}$ with $\left(x_{1}, \ldots, x_{j}, 0, \ldots, 0\right)$ $\in D^{k}$. We begin with a definition.

Definition. Suppose $\varepsilon>0$ and $1 \leq j \leq k$. A sequence $C^{k}, \ldots, C^{j}$; $B^{k}, \ldots, B^{j}$ of subpolyhedra of $R^{k+2}$ is called a $(j, \varepsilon)$-sequence if

(1) each $C^{r}$ is an $(r, \varepsilon)$-collapse in $N_{\varepsilon}\left(H\left(D^{r}\right)\right)$ with $\operatorname{dim} C^{r} \leq r$, and

(2) there is a triangulation $T_{k}$ of $R^{k+2}$ and there are triangulations $T_{r}$ of $\partial B^{r+1}, 1 \leq r<k$, all of mesh $<\varepsilon$ such that $C^{r}$ is a subcomplex of $T_{r}$ and $B^{r}=N\left(C^{r}, T_{r}\right)$.

Note. $B^{r}$ is an $(r+2)$-dimensional PL manifold-with-boundary. 
LemMA 8. For every $\varepsilon>0, \alpha>0$, and $1 \leq j \leq k$ there exists $\delta>0$ with the following property. If $C^{k}, \ldots, C^{j} ; B^{k}, \ldots, B^{j}$ is $a(j, \delta)$-sequence and $X \subset \partial B^{j+1} \cap N_{\delta}\left(h\left(D_{j}^{k}[\alpha]\right)\right)$ is a polyhedron of dimension $\leq j-1$, then there is $a(j, \varepsilon)$-sequence $C_{X}^{k}, \ldots, C_{X}^{j} ; B_{X}^{k}, \ldots, B_{X}^{j}$ such that

(1) $C^{j} \cup X \subset C_{X}^{j}$,

(2) $B_{X}^{r} \cap N_{\delta}\left(h\left(D^{r-1}\right)\right)=B^{r} \cap N_{\delta}\left(h\left(D^{r-1}\right)\right)$ for each $r$, and

(3) if $\operatorname{dim} X<j-1$, then $C_{X}^{j}$ contains $a(j-1, \varepsilon)$-homotopy of $X$.

Proof. The proof is by downward induction on $j$. The case $j=k$, $\operatorname{dim} X=j-1$ is just Lemma 5. Otherwise, if $j=k$ and $\operatorname{dim} X<j-1$, apply Lemma 5 to the track of a $(j-1, \delta)$-homotopy of $X$.

So assume that $j<k$ and that the Lemma is true for $j+1$. Under those assumptions we will prove the Sublemma below. Lemma 8 then follows from the Sublemma together with Lemma 6. The Sublemma supplies the $(j, \delta)$-homotopies we need to apply Lemma 6 with $\Sigma_{\delta}=$ $\left\{\partial B^{j+1} \mid\right.$ there exists a $(j+1, \delta)$-sequence $\left.C^{k}, \ldots, C^{j+1} ; B^{k}, \ldots, B^{j+1}\right\}$.

SUBLEMMA. For every $\varepsilon^{\prime}>0$ there exists $\delta^{\prime}>0$ such that if $C^{k}, \ldots, C^{j}$; $B^{k}, \ldots, B^{j}$ is $a\left(j, \delta^{\prime}\right)$-sequence and $Z \subset \partial B^{j+1} \cap N_{\delta^{\prime}}\left(h\left(D^{j}\right)\right)$ is a compact polyhedron of dimension $\leq j-1$, then there exists $a\left(j, \varepsilon^{\prime}\right)$-sequence $C_{Z}^{k}, \ldots, C_{Z}^{j+1}, C^{j} ; B_{Z}^{k}, \ldots, B_{Z}^{j+1}, B^{j}$ such that $\partial B_{Z}^{j+1}$ contains $a\left(j, \varepsilon^{\prime}\right)$-homotopy of $Z$.

Proof. Use the fact that $B^{j+1}$ is a small regular neighborhood of $C^{j+1}$ to homotope $Z$ to $C^{j+1}$ and then use the $\left(j+1, \delta^{\prime}\right)$-collapse of $C^{j+1}$ to push $Z$ a little further to $N_{\delta^{\prime}}\left(h\left(D_{j+1}^{k}[\gamma]\right)\right)$, where $\gamma$ is a small positive number. Let $\beta(Z)$ denote the image of $Z$ there. Apply Lemma 8 to the $\left(j+1, \delta^{\prime}\right)$-sequence $C^{k}, \ldots, C^{j+1} ; B^{k}, \ldots, B^{j+1}$ with $X=\beta(Z)$. That gives a $\left(j+1, \varepsilon^{\prime}\right)$-sequence $C_{Z}^{k}, \ldots, C_{Z}^{j+1} ; B_{Z}^{k}, \ldots, B_{Z}^{j+1}$ such that $C_{Z}^{j+1}$ contains a $\left(j, \varepsilon^{\prime}\right)$-homotopy of $\beta(Z)$. Furthermore, $B_{Z}^{j+1}$ is the same as $B^{j+1}$ near $h\left(D^{j}\right)$, so we still have $B^{j} \subset \partial B_{Z}^{j+1}$. Thus we can append $C^{j}$ and $B^{j}$ to form the $\left(j, \varepsilon^{\prime}\right)$-sequence $C_{Z}^{k}, \ldots, C_{Z}^{j+1}, C^{j} ; B_{Z}^{k}, \ldots, B_{Z}^{j+1}, B^{j}$.

To finish the proof we must verify that $\partial B_{Z}^{j+1}$ contains a $\left(j, \varepsilon^{\prime}\right)$-homotopy of $Z$. We already know that $C_{Z}^{j+1}$ contains such a homotopy near $h\left(D_{j+1}^{k}[\gamma]\right)$. To get it out into $\partial B_{Z}^{j+1}$, we collapse $C_{Z}^{j+1}$ a little past the track of the homotopy. Once it lies in $B_{Z}^{j+1}-\xi\left(C_{Z}^{j+1}\right)$, we use the product structure given by the fact that $B_{Z}^{j+1} \mathrm{j}$ is a regular neighborhood of $\xi\left(C_{Z}^{j+1}\right)$ to push the track of the homotopy out into $\partial B_{Z}^{j+1}$.

LEMMA 9. For every $\varepsilon>0$ and every $j \leq k$, there exists $a(j, \varepsilon)$-sequence $C^{k}, \ldots, C^{j} ; B^{k}, \ldots, B^{j}$ such that there is a PL map $g^{j-1}: D^{j-1} \rightarrow$ $C^{j}$ with $d\left(h(x), g^{j-1}(x)\right)<\varepsilon$ for every $x \in D^{j-1}$. 
Proof. The proof is by downward induction on $j$. If $j=k$, we apply Lemma 4. Let $X=g^{k-1}\left(D^{k-1}\right)$ for some PL map which is a close approximation to $h \mid D^{k-1}$. By Lemmas 4 and 7 there is a $(k, \varepsilon)$-collapse $C^{k}$ which contains $X$. Take $B^{k}$ to be a small regular neighborhood of $C^{k}$.

Suppose that the Lemma is true for $j+1$. Choose $\delta$ to be the $\delta$ of Lemma 8 and let $C^{k}, \ldots, C^{j+1} ; B^{k}, \ldots, B^{j+1}$ be a $(j+1, \delta)$-sequence as in the statement of the Lemma. Consider $g^{j}: D^{j} \rightarrow C^{j+1}$. By the same construction as in the proof of Lemma 8, there is a map $p: g^{j}\left(D^{j}\right) \rightarrow$ $\partial B^{j+1}$ which is close to the identity and projects $g^{j}\left(D^{j}\right)$ into $\partial B^{j+1}$. Define $g^{j-1}$ to be $p \circ g^{j} \mid D^{j-1}$. Now apply Lemma 8 to the $(j, \delta)$-sequence $C^{k}, \ldots, C^{j+1}, \phi ; B^{k}, \ldots, B^{j+1}, \phi$ with $X=g^{j-1}\left(D^{j-1}\right)$. The sequence $C_{X}^{k}, \ldots, C_{X}^{j} ; B_{X}^{k}, \ldots, B_{X}^{j}$ satisfies the conclusion of Lemma 9 .

Proof of Theorem 1. By Lemma 9 there exists a $(1, \varepsilon)$-sequence for every $\varepsilon^{\prime}>0$. Consider $g^{0}: D^{0} \rightarrow C^{1}$. Since $g^{0}\left(D^{0}\right)$ is just one point, we can find a point $G^{0}\left(D^{0}\right)$ in $\partial B^{1}$ such that $d\left(G^{0}\left(D^{0}\right), h\left(D^{0}\right)\right)<2 \varepsilon^{\prime}$. Extend $G^{0}$ to a PL embedding $G^{1}: D^{1} \rightarrow B^{1}$ using the product structure on $\partial B^{1}$. Then use the $\left(1, \varepsilon^{\prime}\right)$-collapse of $C^{1}$ to stretch $G^{1}$ out to a PL embedding which approximates $h \mid D^{1}$. Now $B^{1} \subset \partial B^{2}$, so we can use the product structure on $\partial B^{2}$ to extend $G^{1}$ to a PL embedding $G^{2}: D^{2} \rightarrow B^{2}$ and then use the $\left(2, \varepsilon^{\prime}\right)$-collapse of $C^{2}$ to stretch $G^{2}$ out to a PL embedding which approximates $h \mid D^{2}$. Then $G^{2}$ is extended to $G^{3}$ : $D^{3} \rightarrow B^{3}$ and so on until we arrive at a PL embedding $G^{k}=g$ which approximates $h$ on all of $D^{k}$.

\section{REFERENCES}

[1] R. H. Bing, Vertical General Position, in Geometric Topology, Lecture Notes in Math., vol. 438, Springer-Verlag, Berlin and New York, 1975, 16-41.

[2] R. T. Miller, Approximating codimension 3 embeddings, Ann. of Math., (2) 95 (1972), 406-416.

[3] L. Montejano, $\beta$-homotopy equivalences have $\alpha$-cross sections, Mem. Amer. Math. Soc., 274 (1983), 1-37.

[4] G. A. Venema, Approximating disks in 4-space, Michigan Math. J., 25 (1978), 19-27.

[5] Approximating topological surfaces in 4-manifolds, Trans. Amer. Math. Soc., 265 (1981), 35-45.

[6] _ Approximating topological embeddings of 3-cells, Abstracts Amer. Math. Soc., $1(1980), 615$.

[7] E. C. Zeeman, Seminar on Combinatorial Topology, Chapters VII and VIII, Mimeographed Notes, Institute des Hautes Etudes Scientifique, Paris, 1963.

Received September 20, 1982 and in revised form July 6, 1984. Research partially supported by National Science Foundation grant number MCS 7902661 A01. 



\section{PACIFIC JOURNAL OF MATHEMATICS EDITORS}

\author{
V. S. VARADARAJAN \\ (Managing Editor) \\ University of California \\ Los Angeles, CA 90024 \\ Herbert Clemens \\ University of Utah \\ Salt Lake City, UT 84112 \\ R. FINN \\ Stanford University \\ Stanford, CA 94305
}

\section{HERMANN FLASCHKA}

University of Arizona

Tucson, AZ 85721

RAMESH A. GANGOLLI

University of Washington

Seattle, WA 98195

VAUGHAN F. R. JONES

University of California

Berkeley, CA 94720

ROBION KIRBY

University of California

Berkeley, CA 94720
C. C. MOORE

University of California

Berkeley, CA 94720

H. SAMELSON

Stanford University

Stanford, CA 94305

HAROLD STARK

University of California, San Diego

La Jolla, CA 92093

\section{ASSOCIATE EDITORS}
R. ARENS
E. F. BECKENBACH
B. H. NEUMANN
F. WOLF
K. YOSHIDA
(1906-1982)

\section{SUPPORTING INSTITUTIONS}

UNIVERSITY OF ARIZONA UNIVERSITY OF OREGON

UNIVERSITY OF BRITISH COLUMBIA UNIVERSITY OF SOUTHERN CALIFORNIA

CALIFORNIA INSTITUTE OF TECHNOLOGY STANFORD UNIVERSITY

UNIVERSITY OF CALIFORNIA

MONTANA STATE UNIVERSITY

UNIVERSITY OF HAWAII

UNIVERSITY OF NEVADA, RENO

UNIVERSITY OF TOKYO

NEW MEXICO STATE UNIVERSITY

UNIVERSITY OF UTAH

OREGON STATE UNIVERSITY

WASHINGTON STATE UNIVERSITY

UNIVERSITY OF WASHINGTON 


\section{Pacific Journal of Mathematics}

\section{Vol. 126, No. $1 \quad$ November, 1987}

John Dauns, Uniform dimensions and subdirect products $\ldots \ldots \ldots \ldots \ldots 1$

William B. Jacob, Quadratic forms over dyadic valued fields. I. The graded

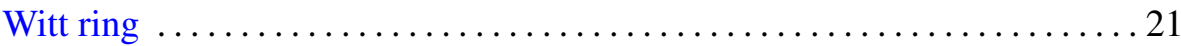

Michael R. Kelly, Minimizing the number of fixed points for self-maps of

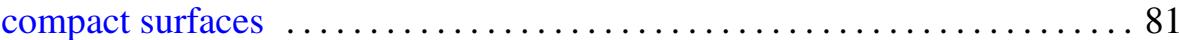

Edward Kissin, On some reflexive operator algebras constructed from two sets of closed operators and from a set of reflexive operator algebras . . 125

Robert Langlands, The Dirac monopole and induced representations . . . . . 145

David A. Stegenga and Kenneth R. Stephenson, Generic covering properties for spaces of analytic functions. II $\ldots \ldots \ldots \ldots \ldots \ldots \ldots 153$

Gerard Alan Venema, Approximating codimension two embeddings of cells 165

Peter Waksman, Determining an analytic function from its distribution of values 